\title{
Mesoporous silica nanoparticles inflame tumors to overcome anti-PD-1 resistance through TLR4-NFKB axis
}

\author{
Mayu Sun, ${ }^{1,2}$ Pengfei Gu, ${ }^{1}$ Yang Yang, ${ }^{1,2}$ Luodan $\mathrm{Yu}^{3}{ }^{2}$ Zheshun Jiang, ${ }^{1}$ Jingquan Li, \\ Yingying Le, ${ }^{2}$ Yu Chen, ${ }^{3}$ Qian Ba, ${ }^{1}$ Hui Wang (D) ${ }^{1,2}$
}

To cite: Sun M, Gu P, Yang Y, et al. Mesoporous silica nanoparticles inflame tumors to overcome anti-PD-1 resistance through TLR4-NFKB axis. Journal for ImmunoTherapy of Cancer 2021;9:e002508. doi:10.1136/jitc-2021-002508

- Additional online supplemental material is published online only. To view, please visit the journal online (http://dx.doi.org/10.1136/jitc2021-002508).

$M S, P G, Y Y$ and $L Y$ contributed equally.

Accepted 02 May 2021

Check for updates

(c) Author(s) (or their employer(s)) 2021. Re-use permitted under CC BY-NC. No commercial re-use. See rights and permissions. Published by BMJ.

For numbered affiliations see end of article.

\section{Correspondence to}

Dr Yu Chen;

chenyu@mail.sic.ac.cn

Dr Qian Ba; qba@shsmu.edu.cn

Dr Hui Wang;

huiwang@shsmu.edu.cn

Yingying Le; yyle@sibs.ac.cn

\section{ABSTRACT}

Background The clinical benefits of antiprogrammed cell death protein 1 (PD-1) therapy are compromised by resistance in immunologically cold tumors. Convergence of immunotherapy and bioengineering is potential to overcome the resistance. Mesoporous silica nanoparticles (MSNs) are considered the most promising inorganic biological nanomaterials for clinical transformation, however, the fundamental influence of MSNs on immunotherapy is unclear. In this study, we aimed to investigate the role of MSNs in tumor resensitization and explore the feasibility of MSNs combined with anti-PD-1 in cancer therapy.

Methods Intrinsic and acquired resistant tumors, as well as spontaneous and secondary tumor recurrence models, were used to evaluate the influence of MSNs and the synergistical effect with anti-PD-1 therapy. The roles of $\mathrm{CD}^{+}$cytotoxic T-lymphocytes (CTLs) and macrophages were assessed in Rag- $1^{-1-}$ mice, ovalbumin/0T-1 TCR transgenic T-cell system, and other blocking mice models. Mechanistic studies were processed by transcriptomics analysis and conducted in primary cells, in vitro coculture systems, and Toll-like receptor 4 (TLR4) knockout mice. Results Both granular and rod-shaped MSNs efficiently overcame tumor resistance with dependence on diameter and aspect ratio. Only once injection of MSNs in prior to anti-PD-1 markedly improved the treatment efficacy, protective immunity, and prognosis. MSNs per se boosted infiltration of CTLs as the early event (days 2-3); and synergistically with anti-PD-1 therapy, MSNs rapidly established a T cell-inflamed microenvironment with abundant high-activated (interferon- $\gamma /$ tumor necrosis factor- $\alpha /$ Perforin/GranzymeB) and low-exhausted (PD-1/lymphocyte-activation gene 3 (LAG-3)/T-cell immunoglobulin and mucin-domain containing-3 (TIM3)) CTLs. Chemokines Ccl5/Cxcl9/Cxcl10, which were produced predominantly by macrophages, promoted MSNs-induced CTLs infiltration. MSNs led to high Ccl5/ Cxcl9/Cxcl10 production in vitro and in mice through regulating TLR4-NF $\kappa B$ axis. Blocking TLR4-NF $\kappa B$ axis in macrophages or CTLs infiltration abrogated MSNs-induced resensitization to anti-PD-1 therapy.

Conclusions MSNs efficiently and rapidly inflame immunologically cold tumors and resensitize them to anti-PD-1 therapy through TLR4-NFKB-Ccl5/Cxcl9/ Cxcl10 axis. MSNs-based theranostic agents can serve as sensitizers for patients with resistant tumors to improve immunotherapy.

\section{BACKGROUND}

Immune checkpoints are important selftolerance regulators in immune system. Under normal physiological conditions, immune checkpoint molecules turn down the activation signals from costimulatory molecules to maintain self-tolerance and prevent autoimmunity. ${ }^{1}$ However, tumor cells can use this mechanism to disrupt $\mathrm{T}$ cell activation and functional status, resulting in $\mathrm{T}$ cell exhaustion and tumor immune escape. ${ }^{2}$ Immune-checkpoint-pathway inhibitors (ICIs), notably antiprogrammed cell death protein 1 antibodies ( $\alpha$ PD-1), show impressive clinical success for various types of cancer. ${ }^{3}$ However, fewer than $50 \%$ of patients derive objective responses to PD-1 blockade. ${ }^{4}$ A major factor to prompt initial ICIs resistance is the so-called 'immunological cold' tumors, characterizing lack of tumor infiltrating lymphocytes. ${ }^{5}$ To destruct cold tumors and further improve therapeutic outcomes and efficacy of ICIs, combination treatments using radiotherapy, chemotherapy, vaccines and other adjuvants have emerged, aimed at reversing resistance, imparting potent therapeutic effects and reducing side effects. ${ }^{6}$

Biomaterials at the nanoscale have attracted substantial attention in biomedicine field. ${ }^{7}$ Nanomaterials feature unique physicochemical properties and biological effects, including tunable shape and size, ease of synthesis, and versatility in surface functionalization. ${ }^{8}$ Since they can be engineered for unique composition and functionality, nanomaterials have been extensively used in medical diagnosis, monitoring, and treatment of cancer. ${ }^{10}$ In addition to functionalized materials, exploration of nanomaterials without specific functional enhancements can provide a fundamental regular pattern for the field of biomaterials. Among these, mesoporous silica nanoparticles (MSNs) are representative of a new generation of 
nanomaterials due to properties such as large surface areas $\left(\sim 1000 \mathrm{~m}^{2} / \mathrm{g}\right)$ and high pore volumes, controllable particle size, excellent biocompatibility/biodegradability, desirable chemical and thermal stabilities, and high loading efficiency of guest molecules. ${ }^{11-13}$ Based on the intrinsic mesoporous nanostructure and high porosity, MSNs have been extensively explored for a variety of biomedical applications, including drugs/gene delivery, bioimaging, and cancer therapy. ${ }^{12}$ Therefore, it is highly worthy for exploring the biological effects of MSNs themselves (without additional functional transformation) on cancer treatment and immunotherapy.

Herein, we report the resensitizing effect of MSNs with different crucial structural parameters on anti-PD-1 immunotherapy. Both granular and rod-shaped MSNs, even only injected once, are able to turn cold tumors hot and overcome resistance to anti-PD-1 therapy in diameterand aspect ratio-dependent manners. Through targeting Toll-like receptor 4 (TLR4)/NF-אB pathway in macrophages, MSNs promote the tumorous infiltration of $\mathrm{CD} 8^{+}$ cytotoxic T-lymphocytes (CTLs). With PD-1 blockade, MSNs synergically activate and reverse the exhaustion of CTLs to result in a long-lasting antitumor immunity. Our findings provide an intriguing insight into the biological impact of MSNs per se on immunotherapy and facilitate the broad applications of nanomedicines in PD-1-targeted cancer immunotherapy.

\section{MATERIALS AND METHODS Chemicals}

Cetyltrimethylammonium chloride (CTAC) was purchased from Sigma-Aldrich. Hexadecyl cetyltrimethylammonium bromide (CTAB), triethanolamine (TEA), tetraethyl orthosilicate (TEOS), ethanol, ammonium hydroxide $\left(\mathrm{NH}_{3} \cdot \mathrm{H}_{2} \mathrm{O}\right)$ and hydrochloric acid $(\mathrm{HCl})$ were obtained from Sinopharm Chemical Reagent Co. Methoxy polyethylene glycol (PEG) silane, MW 2000 (Me-PEG ${ }_{2000}$-silane) was bought from JenKem Technology. All the mentioned chemicals and regents were used directly without further purification. Deionized water (DI water) was used in all experiments.

\section{Synthesis of spherical MSNs with tunable sizes}

Typically, the spherical MSNs were synthesized using a simple soft-templating method. Through adjustment and match of CTAC and TEA, spherical MSNs with varied sizes were synthesized. To synthesize spherical MSNs with diameters of 100,65 and $45 \mathrm{~nm}, 2 \mathrm{~g}$ CTAC was mixed with $0.01 \mathrm{~g}, 0.02 \mathrm{~g}$ and $0.06 \mathrm{~g}$ TEA, respectively, and the mixed solutions were then diluted in $20 \mathrm{~mL}$ DI water by ultrasonic dispersion at room temperature. Then, the solutions were placed in the thermostatic water bath at $80^{\circ} \mathrm{C}, 300 \mathrm{r} / \mathrm{min}$ stirring for $20 \mathrm{~min}$. Afterwards, $1.5 \mathrm{~mL}$ TEOS was added dropwise into the above-mentioned solutions under magnetic stirring $(300 \mathrm{r} / \mathrm{min})$ and the solutions were stirred for another 4 hours to conduct the condensation process in the thermostatic water bath at $80^{\circ} \mathrm{C}$. After cooling down to room temperature, the product was collected by centrifugation $(18000 \mathrm{r} / \mathrm{min}$, $20 \mathrm{~min}$ ) and washed for several times with ethyl alcohol and DI water to remove the residual reactants. Finally, to dislodge the surfactants (CTAC), the prepared MSNs were extracted by circulation reflux with $\mathrm{HCl}$ alcohol solution $(10 \% \mathrm{HCl})$ at $78^{\circ} \mathrm{C}$ for three rounds. To observe the size and morphology of the spherical MSNs, transmission electron microscopy (TEM, JEM-2100F) and scanning electron microscope (SEM, SU9000) were applied.

\section{Synthesis of rod-like MSNs with tunable aspect ratios}

The rod-like MSNs with varied aspect ratios were synthesized based on a single cationic surfactant CTAB and a simple silica source TEOS via a sol-gel reaction. Take rod-like MSNs with a 3:1 length-to-diameter ratio $(\mathrm{L} / \mathrm{D})$, for example, a mixture of CTAB $(0.2 \mathrm{~g}, 8.0 \mathrm{mmol})$, DI water $(70 \mathrm{~mL})$, and ammonium hydroxide $(0.7 \mathrm{~mL}$, $85.5 \mathrm{mmol}$ ) was stirred at $30^{\circ} \mathrm{C}$ for $30 \mathrm{~min}$ at $450 \mathrm{rpm}$ in a $125 \mathrm{~mL}$ wide-mouthed bottle. Then, $1.5 \mathrm{~mL}$ TEOS was added into the above solution dropwise and the condensation process was conducted under stirring for another 4 hours in a water bath at $30^{\circ} \mathrm{C}$. After cooling down to room temperature, the product was gathered by centrifugation $(18000 \mathrm{r} / \mathrm{min}, 15 \mathrm{~min})$ and washed for three times with alcohol, DI water, and alcohol to remove the residual reactants. Each washing round involved a sonication of $20 \mathrm{~min}$ at room temperature. Finally, to remove the surfactants (CTAB), the prepared MSNs were extracted by circulation reflux with $\mathrm{HCl}$ alcohol solution $(10 \% \mathrm{HCl})$ at $78^{\circ} \mathrm{C}$ for three rounds. After washing with alcohol, the MSNs with non-CTAB were dispersed in alcohol for further modification.

To synthesize MSNs with controllable $\mathrm{L} / \mathrm{D}$, the molar quantities of $\mathrm{CTAB}$ and $\mathrm{NH}_{3} \cdot \mathrm{H}_{2} \mathrm{O}$ was modulated. Keeping other parameters constant, when the molar quantities of CTAB and $\mathrm{NH}_{3} \cdot \mathrm{H}_{2} \mathrm{O}$ were $8.0 \mathrm{mM}: 85.5 \mathrm{mM}$, $11.8 \mathrm{mM}: 171.0 \mathrm{mM}$ and $23.6 \mathrm{mM}: 171.0 \mathrm{mM}$, respectively, the L/D have undergone a corresponding change of 3:1, 4:1 and 8.5:1. To determine the $\mathrm{L} / \mathrm{D}$ and observe the nanostructure of the as-prepared MSNs intuitively, TEM and SEM were also used.

\section{Surface PEGylation of MSNs}

To ensure the stability and monodispersity of MSNs in physiological condition, the as-synthesized MSNs were surface-functionalized with PEG. Typically, $10 \mathrm{mg}$ as-prepared MSNs were dispersed into Me-PEG ${ }_{2000}$-silane ethanol solution $(100 \mathrm{~mL}, 0.5 \mathrm{mg} / \mathrm{mL})$ under stirring at $60^{\circ} \mathrm{C}$ overnight. Then, PEG-MSNs was recollected by centrifugation $(18000 \mathrm{r} / \mathrm{min}, 10 \mathrm{~min})$ and rinsed with ethyl alcohol and DI water for several times to remove the redundant $\mathrm{Me}^{-\mathrm{PEG}_{2000}}$-silane. The final obtained PEGylated MSNs were redispersed in PBS solutions and stored under $4^{\circ} \mathrm{C}$ for further use in cellular and animal experiments. 


\section{Mice and cell lines}

C57BL/6 and BALB/C male mice, 4-6 weeks old, were purchased from Shanghai Slac Laboratory Animal and maintained in a pathogen-free vivarium under standard conditions. Rag $1^{-1}$, OT-1 and tumor necrosis factor $(\mathrm{TNFR} 1)^{-/-}$mice, with a C57BL/6 background, were purchased from Shanghai Model Organisms Center. $\mathrm{TLR}^{-/-}$mice with a C57BL/6 background were kindly provided by Professor Yingying Le. ${ }^{14}$ SIBS, CAS. $\beta 2$-microglobulin (B2m)-sgRNA B16F10 tumor cell line was created by using the lentiCRISPRv2 system to deliver sgRNAs against the protein-coding genes, and deletion was confirmed via western blot (Control sgRNA: GCGAGGTATTCGGCTCCGCG, B2m sgRNA: AGTATACTCACGCCACCCAC). B16F10-ovalbumin (OVA) tumor cell line was created by transducing with OVA-expressing lentivirus, and expression was determined via flow cytometry using established anti-mouse SIINFEKL:H-2k ${ }^{\mathrm{b}}$ antibody. B2m-sgRNA B16F10 and B16F10-OVA tumor cells were cultured in DMEM medium. H22 and CT26 tumor cells were cultured in RPMI medium. All mediums were supplemented with $10 \%$ fetal bovine serum, $100 \mathrm{U} / \mathrm{mL}$ penicillin and $100 \mu \mathrm{g} / \mathrm{mL}$ streptomycin. The cell lines used in our study were tested to be free of mycoplasma contamination.

\section{In vivo tumor models}

In the therapeutic experiments, B2m-sgRNA B16F10 $\left(2 \times 10^{5}\right)$ tumor cells were implanted subcutaneously into the left flank of C57BL/ 6 mice. H22 $\left(1.5 \times 10^{6}\right)$ or CT26 $\left(1.5 \times 10^{6}\right)$ tumor cells were implanted subcutaneously into the left flank of BALB/C mice. Tumor-bearing (about $0.5 \mathrm{~cm}$ in diameter) mice were randomized and injected intraperitoneally with PBS vehicle or MSNs prior to antiPD-1 treatment. Anti-PD-1 antibody ( $5 \mathrm{mg} / \mathrm{kg}$, BioXcell) was injected intraperitoneally every two or 3 days. Tumor volume was determined with the formula $1 / 2 a \times b^{2}$ (where $a$ is the long diameter, and $b$ is the short diameter). Mice were killed when the tumor volume reached $3000 \mathrm{~mm}^{3}$. For the tumor rechallenge model, $B 2 m$-sgRNA B16F10 tumor cells $\left(2 \times 10^{5}\right)$ were implanted subcutaneously into the left flank of C57BL/6 mice. The tumors were treated at the indicated time points and resected 4 days after MSNs administration. Then we injected the secondary tumor cells $\left(2 \times 10^{5}\right)$ contralateral to the primary tumor a week after surgery. Mice were observed for tumor growth and survival. To block T cell infiltration, mice were injected with $25 \mu \mathrm{g}$ FTY720 (APExBIO) on day 3 and $5 \mu \mathrm{g}$ on day 4-6 after tumor inoculation. To deplete macrophages, mice were injected with $150 \mu \mathrm{L}$ clodronate liposomes (FormuMax) as the first dose and $100 \mu \mathrm{L}$ clodronate liposomes every 3 days subsequently. To deplete $\mathrm{CD} 8^{+} \mathrm{T}$ cells, mice were injected with $200 \mu \mathrm{g}$ neutralizing anti-CD8 antibody (BioXcell) every 4 days. For adoptive $\mathrm{T}$ cell therapy model, B16F10-OVA tumor cells were implanted subcutaneously into the left flank of $\operatorname{Rag} 1^{-1-}$ mice. OT-1 T cells were isolated from the spleens of OT-1 mice using the EasySep Mouse $\mathrm{CD}^{+}{ }^{+} \mathrm{T}$ Cell
Isolation Kit (Stemcell). When tumor volume was close to $500 \mathrm{~mm}^{3}$, we adoptively transferred OT-1 T cells $\left(8 \times 10^{5}\right)$ into Rag $1^{-/-}$mice-bearing B16F10-OVA tumor. Then, the mice were treated with MSNs or PBS prior to anti-PD-1 treatment. Mice were monitored for tumor growth. For biodistribution of MSNs in mice, B2m-sgRNA B16-F10 tumor-bearing mice were intraperitoneally injected with MSNs, and kidney, liver, lung, spleen and tumor tissue were harvested at 24 hours post-injection and dissolved in aqua regia for ICP-MS measurement. The content of $\mathrm{Si}$ in each organ was expressed as percentage of the injected dose per gram of tissue $(\% \mathrm{ID} / \mathrm{g})$.

\section{Antibodies and reagents}

Anti-PD-1 antibody (clone: RMP1-14) and isotype control were obtained from BioXcell. The fluorochrome-labeled antimouse antibodies used for flow cytometry measurements were Fixable Viability Stain 780 (565388; BD Biosciences); PE-CD45 (553081; BD Biosciences); FITCCD45 (553080; BD Biosciences); BV510-CD45 (563891; BD Biosciences); FITC-CD3e (553061; BD Biosciences); BV421-CD8 (563898; BD Biosciences); BV510-CD8 (563068; BD Biosciences); APC-CD4 (553051; BD Biosciences); FITC-CD4 (553080; BD Biosciences); PE-Cy7CD11b (552850; BD Biosciences); FITC-Ly6G (551460; BD Biosciences); APC-Ly6C (560595; BD Biosciences); BV421-F4/80 (123132; Biolegend); PerCP-Cy5.5-I-A/I-E (562363; BD Biosciences); PE-natural killer (NK)-1.1 (553165; BD Biosciences); PE-SIINFEKL:H-2kb (141603; BD Biosciences); APC-interferon- $\gamma$ (IFN- $\gamma$ ) (554413; BD Biosciences); PE-PD-1 (109103; Biolegend); BV421-TIM3 (119723; Biolegend); PE-Cy7-LAG-3 (125226; Biolegend); PE-Cy7-Ccl5 (149106; Biolegend); PE-Cxcl9 (515604; Biolegend); CD16/32 (553141; BD Biosciences); APCCD206 (141707; Biolegend). The anti-mouse antibodies used for immunobloting were anti-B2m antibody (ab75853; Abcam); anti-TLR4 antibody (14358; CST); anti-TRAF6 antibody (ab33915; Abacam); anti-MYD88 antibody (4283; CST); anti-TNFR1 antibody (ab19139; Abcam); anti-TRADD antibody (3694; CST); anti-RIP1 antibody (3493; CST); anti-TRAF2 antibody (4724; CST); anti-TAK1 antibody (5206; CST); anti-Phospho-TAK1 antibody (9339; CST); anti-IKK $\alpha / \beta$ antibody (2370; CST); anti-Phospho-IKK $\alpha / \beta$ antibody (2697; CST); anti-

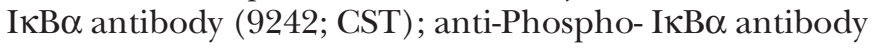
(2859; CST); anti-Phospho-NF-кB P65 antibody (3033; CST); anti-NF-kB P65 antibody (4764; CST); anti-GAPDH antibody (2118; CST); Anti-rabbit IgG antibody (5127; CST).

\section{Analysis of infiltrating immune cells by flow cytometry}

Mice were sacrificed by cervical dislocation under anesthesia using $6 \%$ chloral hydrate. Tumor tissues were collected and cut into small pieces, then dissociated into single-cell suspensions with mouse tumor dissociation kits (Miltenyi Biotec) following the manufacturer's instructions. After filtering through a $70 \mu \mathrm{m}$ Falcon cell strainer, cells suspensions were blocked with antimouse 
CD16/32 antibody for 15 min and stained with Fixable Viability Stain (1:1000, BD Biosciences) for $10 \mathrm{~min}$ to exclude dead cells. After washing, cells were stained with indicated antibodies for $30 \mathrm{~min}$ in the dark. The cells were then washed twice with PBS and analyzed by BD Fluorescence Activated Cell Sorting (FACS) Aria II (BD Biosciences, San Jose, California, USA). For intracellular cytokine, tumor-infiltrating leukocytes were enriched using an Optiprep (Sigma) density gradient and restimulated at $37^{\circ} \mathrm{C}$ with Leukocyte Activation Cocktail plus BD GolgiPlug (BD Biosciences) for 4 hours, followed by fixation and permeabilization using the Cytofix/Cytoperm Fixation/Permeabilization kit (BD Biosciences). For intranuclear staining, cells were permeabilized with the Foxp3 Transcription Factor Staining Buffer Kits (eBioscience). Cell suspensions were stained as described above.

\section{Quantitative real-time PCR}

Total RNA was extracted with the TRIzol reagent (Invitrogen) and reverse-transcribed into cDNA. qRT-PCR was performed on an ABI7900HI (Applied Biosystems). Target gene expression was normalized to Actin. Primers were shown in online supplemental table 1.

\section{Protein extraction and immunoblotting}

Cells were lysed with RIPA buffer supplemented with freshly added protease inhibitor cocktail (Roche). After incubation on ice for $30 \mathrm{~min}$, cell lysates were centrifuged at $12000 \mathrm{rpm}$ for $20 \mathrm{~min}$ at $4^{\circ} \mathrm{C}$. Subsequently, protein concentrations in the supernatant were determined by Pierce BCA Protein Assay Kit (Thermo Fisher Scientific). Supernatant was heated to $95^{\circ} \mathrm{C}$ in $6 \times$ Protein Loading Buffer (TransGen) for $10 \mathrm{~min}$. Proteins were separated by SDS-PAGE and transferred to PVDF membrane (Millipore). Membranes were blocked in Tris-buffered saline plus $0.1 \%$ Tween 20 (TBST) containing $5 \%$ nonfat dry milk for 1 hour and probed with indicated antibodies overnight. Membranes were washed with TBST for three times and incubated with Horseradish peroxidase (HRP)-conjugated secondary antibodies for 2 hours. The bands were detected with Tanon-5200 Chemiluminescent Imaging System (Tanon Science \& Technology).

\section{$T$ cells migration assay}

$\mathrm{CD}^{+} \mathrm{T}$ cells were isolated from $\mathrm{C} 57 \mathrm{BL} / 6$ mice. Bone marrow-derived macrophages (BMDMs) were obtained by flushing the femurs of mice and cultured for 1 week in Iscove's Modified Dulbecco's Medium containing $20 \mathrm{ng} /$ mL M-CSF. $1 \times 10^{6}$ CTLs in complete media were loaded into the top chamber of Transwell inserts $(5.0 \mu \mathrm{m}$ pore size, Corning). The lower chamber was filled with conditioned medium from Raw 264.7 cells or BMDMs after treated with MSNs for 12 hours. Plates were incubated for 3 hours, the contents of the bottom chamber were collected, and the CTLs were counted.

\section{Microscale thermophoresis}

Microscale thermophoresis (MST) measurements were performed on a Monolith NT.115 (NanoTemper).
FITC-labeled MSNs were diluted with PBS to $0.2 \mathrm{mg} / \mathrm{mL}$. Human TLR4 (1478-TR-050; R\&D Systems) was diluted into a series of concentrations and mixed with the same volume of MSNs. Then samples were loaded into capillaries. The thermophoresis was monitored at $40 \%$ MST power. Data were analyzed and fit to the KD model using MO. Affinity software (V.2.3).

\section{Transcriptomic analysis}

Mice bearing $B 2 m$-sgRNA B16F10 tumor were treated with PBS vehicle or MSNs prior to anti-PD-1 treatment. After 11 days, mice were euthanized and the tumor tissues were collected for RNA extraction and transcriptome sequencing. Sequencing libraries were generated using NEBNext UltraTM RNA Library Prep Kit for Illumina (NEB, USA) following manufacturer's recommendations. The library preparations were sequenced on an Illumina Novaseq platform (Novogene, Beijing, China) and $150 \mathrm{bp}$ paired-end reads were generated. Reads were mapped to the reference genome using Hisat2 v2.0.5. FeatureCounts v1.5.0-p3 was used to count the reads numbers of each gene. And FPKM of each gene was calculated for subsequent expression analysis. Gene correlation was determined by Spearman's correlation coefficients. Gene specific and transcriptional factor enrichment were analyzed through Metascape. ${ }^{15}$ Kyoto Encyclopedia of Genes and Genomes (KEGG) pathway enrichment and Gene Set Enrichment Analysis (GSEA) were performed by the R package 'clusterProfiler'.

\section{Statistical analysis}

All values were presented as the means \pm SEM. The data were analyzed by using GraphPad Prism V.7 (GraphPad Software). Statistical significance was determined through unpaired Student's t-test or log-rank (Mantel-Cox) test. The differences in tumor growth were analyzed at the end time point. A $\mathrm{p}<0.05$ was considered significant.

\section{RESULTS \\ MSNs sensitize resistant tumors to anti-PD-1 therapy and improve survival}

The properties such as size, shape, and surface functionalization of nanoparticles influence the biological effects in vivo. To assess the immunological effects and the influences of properties, MSNs with tunable sizes and shapes were synthesized. Spherical MSNs with diameters (ranging from ca. $45-100 \mathrm{~nm}$ ) were facilely synthesized with excellent dispersity and evident mesoporous structure by regulating the mass ratio of TEA as the alkaline catalyst and CTAC as the structural-directing agent (figure 1A). Rodlike MSNs with varied $\mathrm{L} / \mathrm{D}$ were synthesized by altering the dosage of surfactant (CTAC) during the synthesis process, which were directly observed as spirally mesoporous with homodispersed nanostructure (figure 1B). The preparation, nanostructure and composition made these MSNs suitable for evaluation of size-dependent and morphology-dependent effects. 
A

A

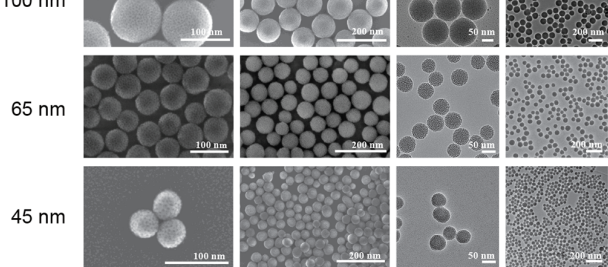

C
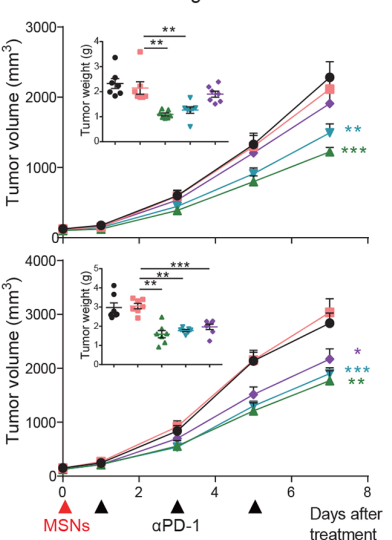

F
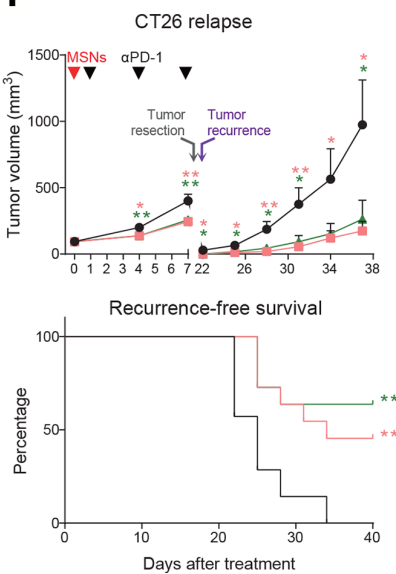

D $\quad$ H22
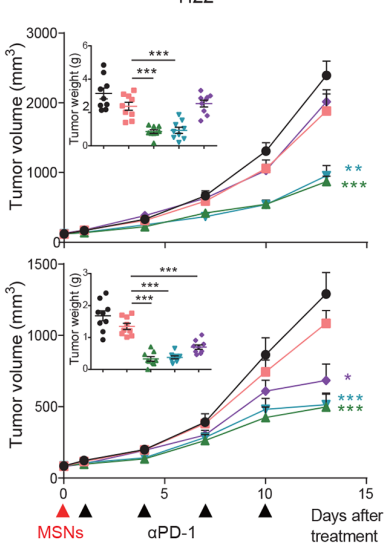

G
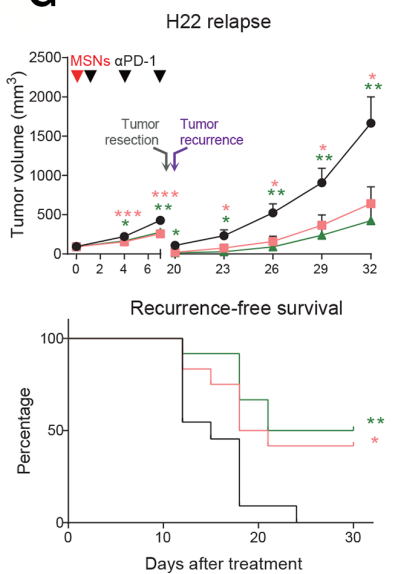

B

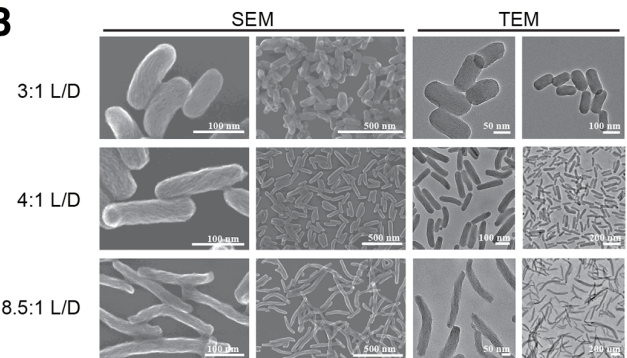

E

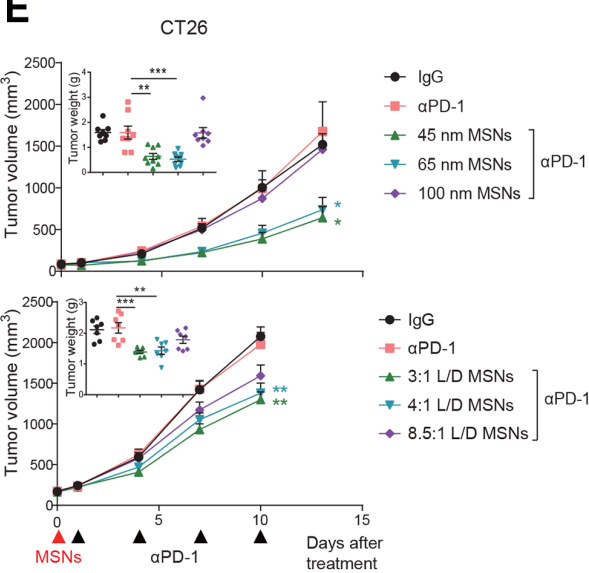

H
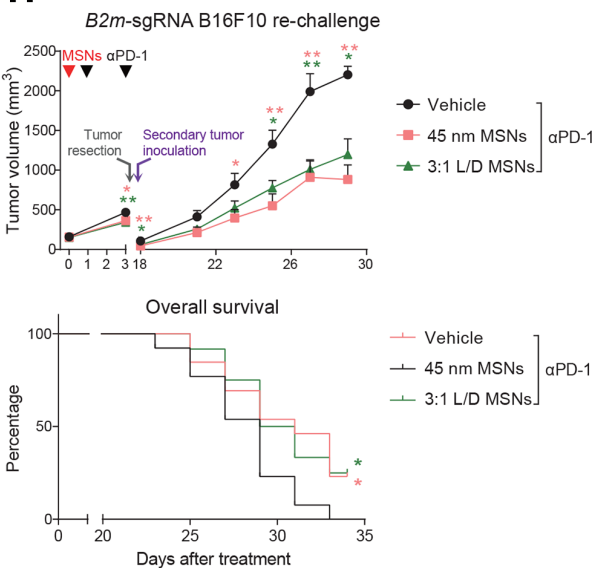

Figure 1 MSNs overcome resistance and prevent relapse of $\alpha$ PD-1-resistant tumors. (A) SEM and TEM images of spherical MSNs with diameters of 100, 65 and $45 \mathrm{~nm}$. (B) SEM and TEM images of rod-like MSNs with aspect ratios of 3:1, 4:1, 8.5:1. (C-E) Tumor growth and tumor weights of B2m-sgRNA B16F10 (C), H22 (D) and CT26 (E) tumor-bearing mice treated with different MSNs treatment (top: sizes; bottom: L/Ds) prior to $\alpha P D-1 .(F, G)$ Growth of recurrent tumors (top) and recurrence-free survival (bottom) of tumor-bearing mice after indicated treatments. (H) Growth of rechallenged tumors (top) and overall survival (bottom) of B2m-sgRNA B16F10 tumor-bearing mice after indicated treatments. Error bars represent mean \pm SEM ( $n>6$ per group in $\mathrm{C}-\mathrm{H}$ ). $\mathrm{P}$ value for tumor measurement was calculated by unpaired Student's t-test. $\mathrm{P}$ value for survival curve was calculated by the log-rank (Mantel-Cox) test. $\left({ }^{\star} \mathrm{P}<0.05,{ }^{* \star} \mathrm{p}<0.01,{ }^{\star \star \star} \mathrm{p}<0.001\right)$. MSNs, mesoporous silica nanoparticles; PD-1, programmed cell death protein 1 ; TEM, tumor microenvironment.

To investigate the potential influence of MSNs on immunotherapy-resistant tumors, we employed syngeneic mouse models. First, we established acquired $\alpha$ PD-1resistant tumors through impairing $B 2 m$ in $\mathrm{B} 16 \mathrm{~F} 10$ melanoma (B2m-sgRNA B16F10, online supplemental figure $1 \mathrm{~A}, \mathrm{~B})$, and found that pretreatment with MSNs prior to $\alpha$ PD-1, even only once, significantly restored sensitivity to PD-1 blockade and eliminated resistant tumors in size- or L/D-dependent manner (figure 1C and online supplemental figure 1I). Smaller size and L/D MSNs yielded the most robust therapeutic effect across all groups (53\% for $45 \mathrm{~nm}$ and $47 \%$ for 3:1 L/D). Second, we used two intrinsic resistant tumors, H22 hepatocellular carcinoma and CT26 colorectal carcinoma, ${ }^{16}{ }^{17}$ for further validation. These tumors were also resensitized to $\alpha \mathrm{PD}-1$ by all MSNs except for spherical $100 \mathrm{~nm}$ particles 
(figure 1D,E and online supplemental figure 1I). Therefore, MSNs overcame resistance to PD-1 blockade in both acquired and intrinsic resistant tumors. Notably, MSNs without immunotherapy (plus IgG) did not elicit antitumor response (online supplemental figure 1C-I), suggesting that MSNs induced the anti-PD-1 sensitization rather than a direct therapeutic efficacy. Additionally, no obvious differences in body weight were observed among groups (online supplemental figure 2).

We next investigated whether MSNs could induce longlasting antitumor immunity through two relapse models. In spontaneous relapse model, after MSNs and $\alpha \mathrm{PD}-1$ treatment, tumors were resected and the recurrence was monitored. Pretreatment with MSNs significantly reduced postoperative recurrence and the growth of recurrent tumors even if no longer therapy (figure 1F,G), suggesting that MSNs plus $\alpha \mathrm{PD}-1$ enhanced immune memory effect effectively. In rechallenged model, we inoculated secondary tumors contralaterally after MSNs/ $\alpha \mathrm{PD}-1$ treatment and surgical removal of primary foci. The growth of secondary $B 2 m$-sgRNA B16F10 tumors were suppressed in MSNs plus $\alpha \mathrm{PD}-1$ groups, which achieved a significantly longer overall survival (figure $1 \mathrm{H}$ ). These results demonstrate that MSNs enhanced the systemic antitumor immune response during PD-1 blockade therapy and improved survival.

\section{MSNs overcome tumor resistance in a CTLs-dependent manner}

To characterize the causality for MSNs-induced resensitization to anti-PD-1, we first measured biodistribution of MSNs in mice bodies. At 24 hours after injection, MSNs preferentially accumulated in tumors, however, there was no correlation between distribution amount and therapeutic efficiency (online supplemental figure 3 ). Then, we analyzed the immune composition in tumor microenvironment (TME) after treatment. In both intrinsic and acquired resistant tumors, MSNs (except $100 \mathrm{~nm}$ ) plus $\alpha \mathrm{PD}-1$ treatment showed greater infiltration of immune cells $\left(\mathrm{CD} 45^{+}\right)$ compared with $\alpha \mathrm{PD}-1$ treatment alone (online supplemental figure $4 \mathrm{~A}-\mathrm{C}$ ). The proportion of $\mathrm{CD}^{+}$CTLs increased significantly under treatment with both spherical and rod-like MSNs combined with $\alpha \mathrm{PD}-1$ in all types of tumor models (figure 2A and online supplemental figure $4 \mathrm{D}-\mathrm{F})$. Moreover, MSNs-induced elevation of $\mathrm{CD} 45^{+}$and $\mathrm{CD}^{+}$cells was positively correlated with the therapeutic effect, indicating that $\mathrm{CD} 8^{+}$CTLs played important roles in this process. Other immune cells, including $\mathrm{CD} 4^{+} \mathrm{T}$ cells, granulocytic myeloid-derived suppressor cells (MDSCs), monocytic MDSCs, macrophages, dendritic cells, and NK cells, did not always show a consistent trend under treatment with MSNs coupled with $\alpha \mathrm{PD}-1$ (figure 2A and online supplemental figure 4D-F).

In order to explore the involvement of CTLs in MSNsinduced antitumor effect, we adopted recombinaseactivating gene 1-deficient $\left(\operatorname{Rag} 1^{-/}\right)$mice, which lack mature $\mathrm{T}$ and $\mathrm{B}$ cells, ${ }^{18}$ and set up an adoptive T cell therapy model with OVA-specific CTLs from OT-1 TCR transgenic mice
(OT-1 CTLs) (figure 2B). B16F10 cells stably expressing chicken OVA, which are recognizable by $\mathrm{T}$ cells, were inoculated in this mice model. We found that, in $\operatorname{Rag} 1^{-/}$mice, pretreatment with MSNs can no longer overcome tumor resistance (figure 2C). However, adoptive transfer of OT-1 CTLs conferred Rag1 ${ }^{-/}$mice antitumor immunity and restored the resensitizing effect of MSNs (figure 2D). Consistently, the resensitizing effect of MSNs on CT26 tumor was abolished by $\mathrm{CD}^{+} \mathrm{T}$ cells depletion through anti-CD8 antibody (online supplemental figure 5). These results indicate that CTLs essentially mediated the antitumor immune responses induced by MSNs.

\section{MSNs and $\alpha \mathrm{PD}-1$ synergistically establish a T cell-inflamed TME at the beginning of treatment}

Considering that $\alpha \mathrm{PD}-1$ or MSNs alone did not show therapeutic efficacy in resistant tumors (figure 1 and online supplemental figure 1), we explored the potential synergistic effect of MSNs and $\alpha$ PD-1 on TME specified as tumorous CTLs. Early stage (day 2-3 after treatment) was focused to reveal the potential causative factors. We found that: (1) Consistent with treatment tolerance, neither the infiltration (total), cytotoxicity $\left(\mathrm{IFN}-\gamma^{+}\right)$, nor exhaustion $\left(\mathrm{LAG}_{-} 3^{+}\right.$, TIM- $3^{+}$) of CTLs were influenced by $\alpha \mathrm{PD}-1$ alone (figure 3A). PD- $1^{+}$CTLs were reduced after $\alpha$ PD-1 treatment, which is in line with the effect of PD-1 antibody. (2) In contrast with $\alpha$ PD-1, although cytotoxicity and exhaustion were not affected, MSNs alone significantly increased the infiltrated CTLs in tumors (figure 3B), indicating that MSNs per se can inflame TME by promoting tumorous CTLs infiltration. (3) Furthermore, combined treatment resulted in a thorough CTLs activation with dramatically increased infiltration and cytotoxicity, while, reduced exhaustion (figure 3C). These results indicate that MSNs and $\alpha \mathrm{PD}-1$ synergistically prompted CTLs infiltration and activation in TME at the beginning of treatment.

To further illustrate the functional status of CTLs, we isolated CTLs from early-stage tumors (day 3 after treatment). On one hand, the levels of $\mathrm{T}$ cell activation markers (CD27, 4-1BB and OX40), proliferation marker (Ki67), and functional factors (Granzyme-B, Perforin, INF- $\gamma$, and TNF- $\alpha$ ), were significantly increased by pretreatment with MSNs (figure 3D). On the other hand, transcriptional factors that establish and sustain $\mathrm{T}$ cell exhaustion (Tox, FoxO1, FoxP1, etc) were decreased under MSNs pretreatment (figure 3D). These results demonstrate that even at early stage of combine treatment, MSNs can inflame TME characterized with abundant high-activated and lowexhausted CTLs.

Since MSNs per se promoted CTLs infiltration at early stage, we further validated the role of CTLs infiltration in TME inflaming and antitumor responses. We utilized S1PR1 inhibitor, FTY720, to block T lymphocytes trafficking (figure 3E). ${ }^{19}$ Strikingly, the resensitizing effect of MSNs on resistant tumors to $\alpha \mathrm{PD}-1$ was completely abrogated in the context of FTY720 (figure 3E,F). Therefore, we conclude that MSNs, through promoting CTLs 


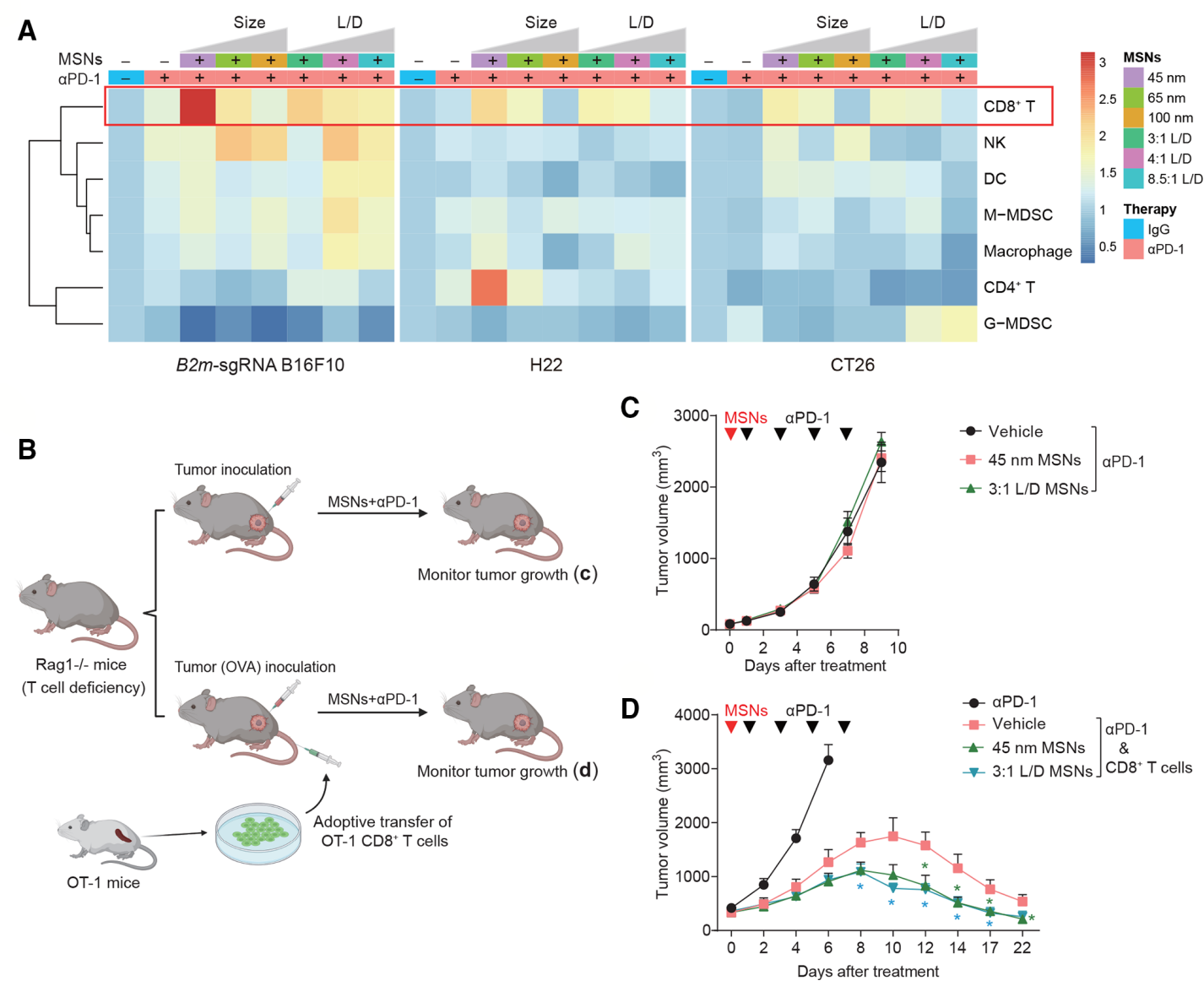

Figure 2 MSNs resensitize tumors to $\alpha \mathrm{PD}-1$ through CTLs. (A) Heatmap showing the proportion of tumor-infiltrating $\mathrm{CD}^{+} \mathrm{T}$ cells, NKs, DCs, M-MDSCs, macrophages, $C D 4^{+} T$ cells and G-MDSCs in the Tme after indicated treatments. (B) Scheme of tumor therapy in T cell-deficiency Rag $1^{-1-}$ mice and T cell-regained mice by transferring. (C) Growth of B2m-sgRNA B16F10 tumors in Rag $1^{-/-}$mice after indicated treatments. (D) Growth of B16F10-OVA tumors in Rag $1^{-/-}$mice with adoptive transfer of OT-1 CTLs. Error bars represent mean \pm SEM $(n>5$ per group in $(C, D))$. $P$ value was calculated by unpaired Student's t-test $\left({ }^{*} \mathrm{P}<0.05,{ }^{* *} \mathrm{P}<0.01,{ }^{* *} \mathrm{p}<0.001\right)$. CTLs, cytotoxic T-lymphocytes; DCs, dendritic cells; G-MDSCs, granulocytic myeloid-derived suppressor cells; M-MDSCs, monocytic MDSCs; MSNs, mesoporous silica nanoparticles; NKs, natural killer; OVA, ovalbumin; PD-1, programmed cell death protein 1.

infiltration, inflamed TME and overcame anti-PD-1 resistance synergistically with $\alpha$ PD-1.

\section{MSNs drive T cell-recruitment chemokines production in TAMs to promote CTLs infiltration}

To explore how MSNs promoted tumorous CTLs infiltration, we analyzed chemokines trafficking immune cells in tumor tissues, and found that $\mathrm{T}$ cell-recruitment chemokines (Ccl5, Cxc19 and Cxcl10) were significantly increased after treatment with spherical or rod-like MSNs plus $\alpha \mathrm{PD}-1$ (figure $4 \mathrm{~A}$ ). To depict the effector cell types in TME, chemokine-expressing cell populations were isolated from tumor tissues and the compositions were identified. As shown in figure 4B and online supplemental figure 6), macrophages $\left(\mathrm{CD} 11 \mathrm{~b}^{+} \mathrm{F} 4 / 80^{+}\right)$dominantly engaged chemokines production, while other immune cells and non-immune cells only accounted for the minority. The influences of MSNs on chemokines in these cell types were further determined. At the beginning of treatment (day 2), in sorted macrophages (TAMs) rather non-immune cells, MSNs pretreatment significantly upregulated the expression of Ccl5, Cxc19 and Cxcl10 even without $\alpha \mathrm{PD}-1$ therapy (figure $4 \mathrm{C}, \mathrm{D}$ ), not only confirming the infiltration-promoting effect of MSNs per se, but also suggesting the mediating role of TAMs in this process.

To confirm the mediation of macrophages for MSNsinduced $\mathrm{T}$ cell recruitment, we performed in vitro coincubation assays (figure 4E). After treatment with MSNs, macrophages were used to examine chemokine expressions, and the conditional medium were harvested for $\mathrm{T}$ cell recruitment assay. Following MSNs treatment, the expression of Ccl5, Cxcl9 and Cxcl10 in macrophages was significantly elevated (figure $4 \mathrm{~F}$ ); and macrophage medium-mediated $\mathrm{CD} 8^{+} \mathrm{T}$ cell recruitment was also markedly enhanced (figure 4G). We further determined the role of macrophages in MSNs-induced CTLs infiltration and antitumor effect in vivo. Clodronate liposomes can specifically deplete blood monocytes and tissue macrophages without affecting other immune cells. ${ }^{20}$ Macrophage depletion in mice by clodronate liposomes resulted 

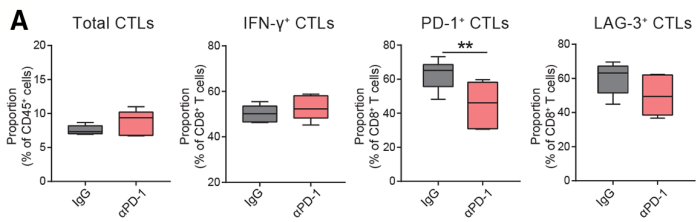

B Total CTLS
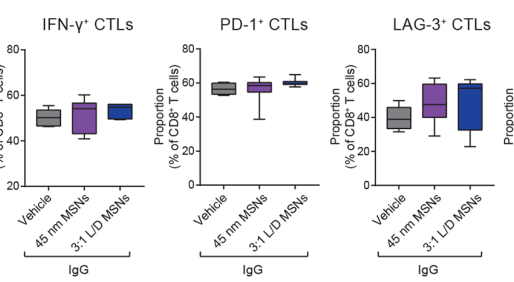

PD-1+ CTLS

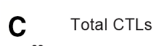

IFN-Y+ CTLS
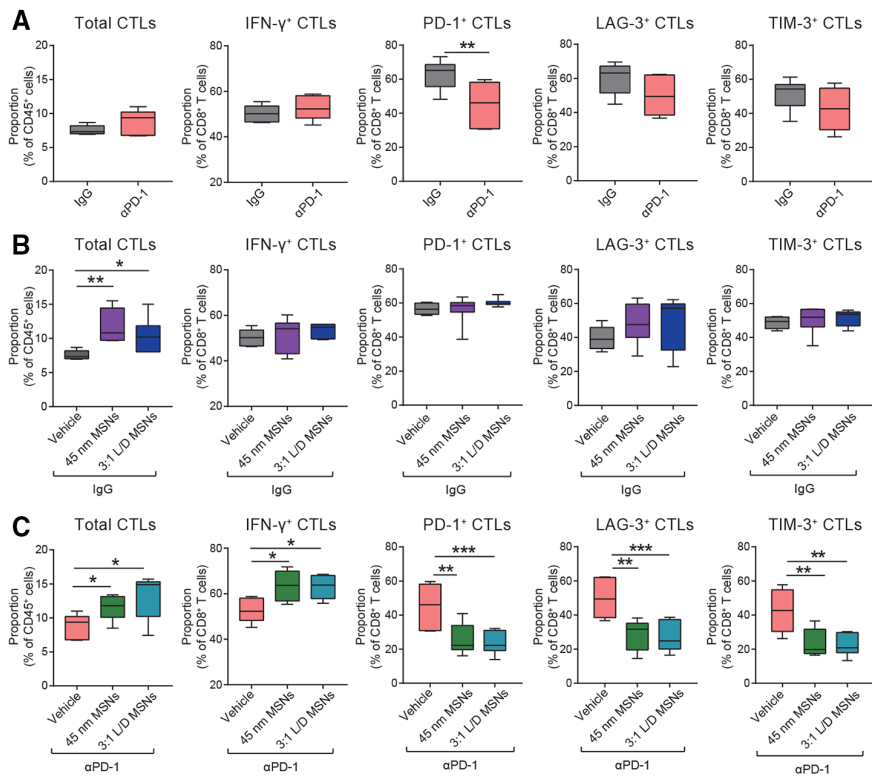

TIM-3+ CTLS

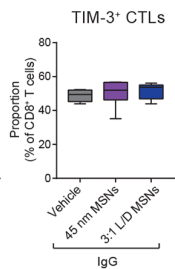

TIM- $3^{+}$CTLS
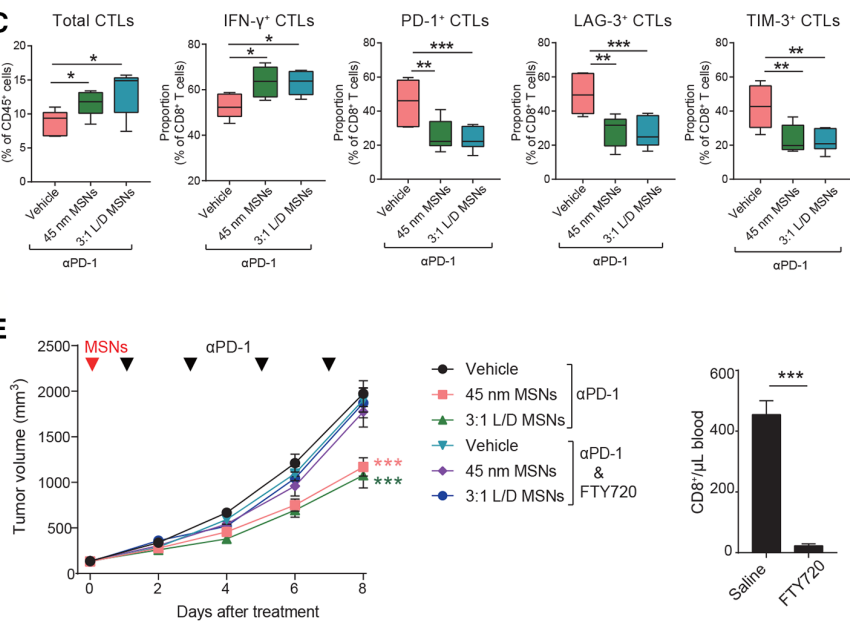

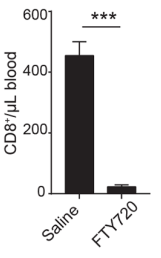

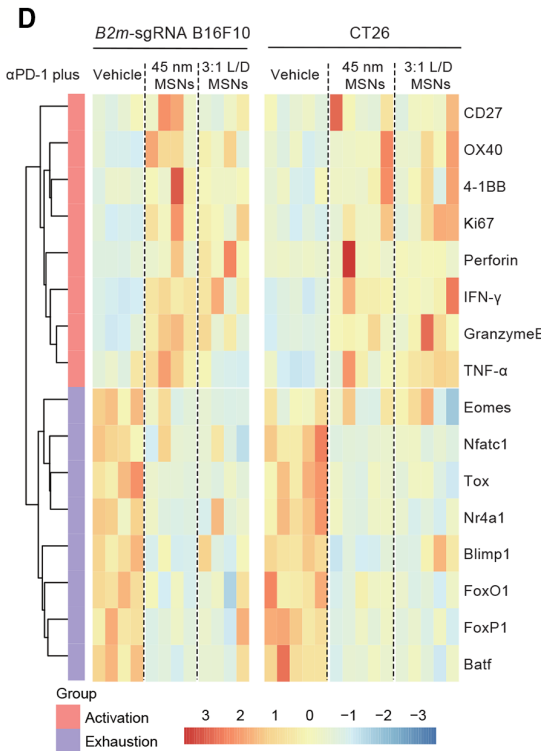

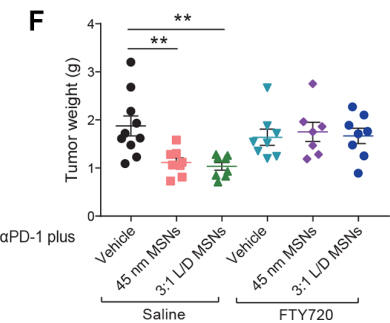

Figure 3 MSNs drive CTLs infiltration to promote CTLs activation synergistically with $\alpha P D-1$ at the beginning of treatment. (A-C) On day 2-3 after indicated treatments, the proportion of total CTLs and INF- $\gamma$, PD-1, LAG-3 and Tim-3 positive CTLs in B2m-sgRNA B16F10 tumor tissues was quantified by flow cytometry. (D) On day 3 after indicated treatments, the mRNA expression in CTLs isolated from B2m-sgRNA B16F10 or CT26 tumor tissues was shown. (E, F) Tumor growth (E) and tumor weights $(\mathrm{F})$ of $B 2 m$-sgRNA B16F10 tumor-bearing mice treated with FTY720 to block T cell infiltration. Control mice received saline. Flow cytometry analysis quantified the numbers of CTLs collected from blood 3 days postinitial FTY720 administration. Error bars represent mean \pm SEM ( $n>6$ per group in E). P value was calculated by unpaired Student's t-test $\left({ }^{*} p<0.05,{ }^{* *} p<0.01\right.$, $\left.{ }^{* \star *} \mathrm{p}<0.001\right)$. CTLs, cytotoxic T-lymphocytes; INF- $\gamma$, interferon- $\gamma$; MSNs, mesoporous silica nanoparticles; PD-1, programmed cell death protein 1 ; TNF $\alpha$, tumor necrosis factor- $\alpha$.

in a complete abrogation of the resensitizing effect of MSNs (figure $4 \mathrm{H})$. Importantly, tumorous $\mathrm{CD}^{+} \mathrm{T}$ cells infiltration (CD8a), and chemokines (Ccl5, Cxc19, and Cxcl10) were no longer induced by MSNs in macrophagedepleted mice (figure 4I). The same phenomenon was observed in adoptive $\mathrm{T}$ cell therapy model (online supplemental figure 7), confirming the prerequisite of macrophages for MSNs-promoted CTLs infiltration and tumor sensitization.

We further determined whether MSNs could drive macrophage polarization toward M1 in the TME. In $B 2 m-$ sgRNA B16F10 and CT26 tumors, MSNs pretreatment promoted a shift of macrophages from M2 toward M1 type and increased the ratio of M1/M2 macrophages (online supplemental figure 8A,B). In H22 model, MSNs-treated tumor tissues showed a decline in M2 marker (arginase 1 (Arg-1)) and an increase in M1 marker (inducible nitric oxide synthase (iNOS)) (online supplemental figure 8C). These results indicate that MSNs regulate macrophages in terms of activity rather than quantity.
These results suggest that MSNs drove the state transition of macrophages and macrophage-intrinsic chemokines expression promoted CTLs infiltration.

\section{MSNs activate NF- $\mathrm{kB}$ signaling in tumors for production of $\mathrm{T}$ cell-recruitment chemokines}

To investigate the mechanism of action that MSNs drove $\mathrm{T}$ cell-recruitment chemokines, we performed transcriptomics (RNA-seq) analysis using tumor tissues after MSNs and $\alpha$ PD- 1 treatments. Across all samples in different groups, there was strong correlation among the expression of $\mathrm{Ccl} 5$, Cxc19, and Cxcl10 (figure 5A), indicating that these chemokines showed the same pattern. Considering that same pattern genes generally share the regulatory module, we thus focused on genes with significantly positive association with Ccl5 $(\mathrm{p}<0.01)$ (figure 5B). Through cell-specific enrichment, macrophages were identified as the most highly responsive cell type (figure 5C), further supporting the above results that macrophages were the major source of $\mathrm{T}$ cell-recruitment chemokines. 


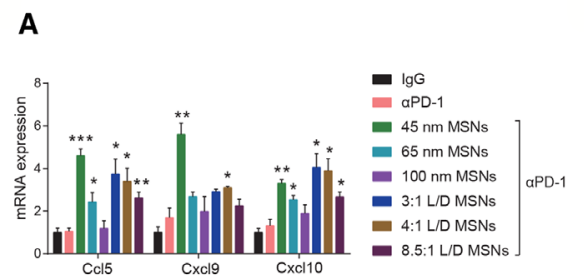

C Intratumoral macrophages

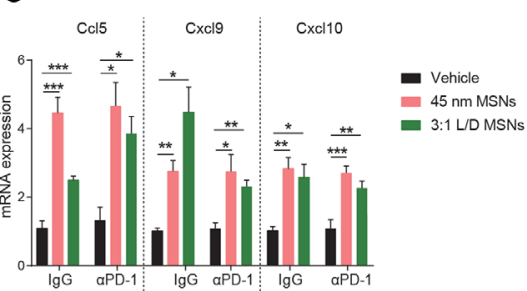

E

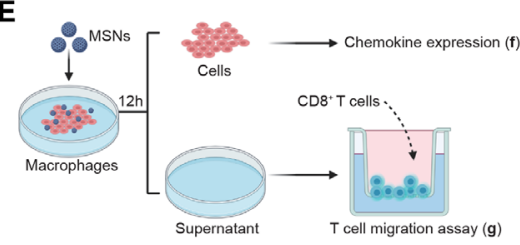

H

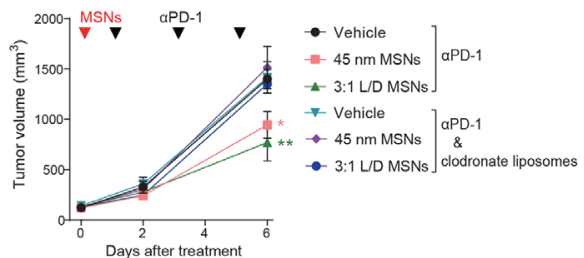

$B_{3}$

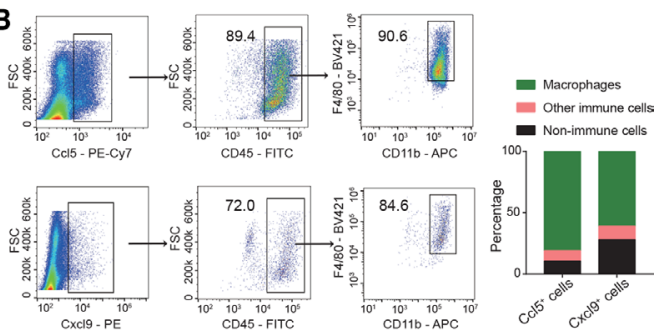

D

D Intratumoral non-immune cells
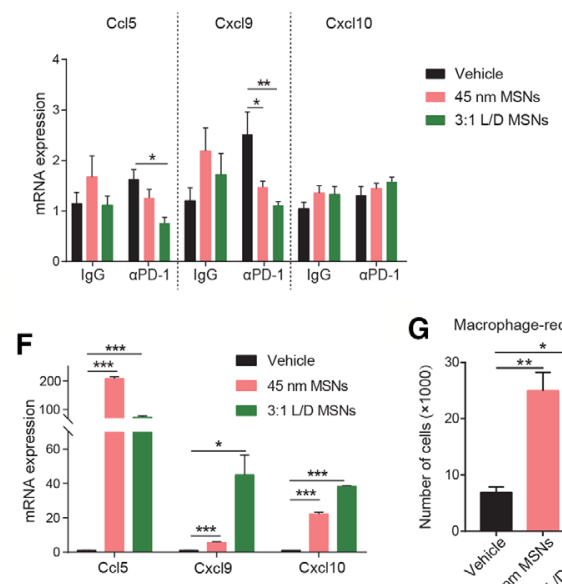

G Macrophage-recruited $\mathrm{T}$ cells

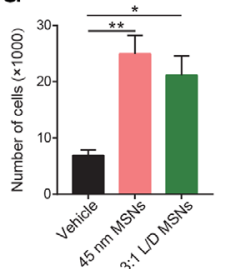

I

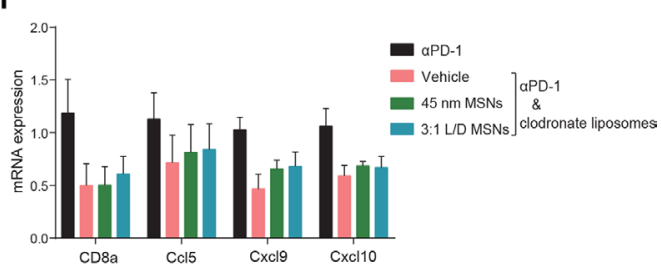

Figure 4 MSNs promote the production of T cell-recruitment chemokines in macrophages. (A) The mRNA expression of CCL5, CXCL9, and CXCL10 in B2m-sgRNA B16F10 tumor tissues after indicated treatments was determined by qRT-PCR. (B) The major cell types that produced CCL5 and CXCL9 in B2m-sgRNA B16F10 tumor tissues were analyzed by flow cytometry. the right graph quantified the percentage of CCL5 and CXCL9 producing cell types. (C, D) The mRNA expression of CCL5, CXCL9 and CXCL10 in intratumoral macrophages (CD11b+F4/80+) (C) and non-immune cells (CD45-) (D) isolated from B2m-sgRNA B16F10 tumor tissues was determined by qRT-PCR. (E) Scheme of in vitro macrophages treatment and coincubation. RAW 264.7 cells were treated by $100 \mu \mathrm{g} / \mathrm{mL}$ MSNs for 12 hours. Cells were collected for gene expression assay, and supernatant was collected for T cell recruitment assay. (F) The mRNA expression of CCL5, CXCL9 and CXCL10 in RAW 264.7 cells after MSNs treatments was determined by qRT-PCR. (G) Quantification of migrated T cells recruited by the supernatant of macrophages after MSNs treatments. $(\mathrm{H})$ Tumor growth of B2m-sgRNA B16F10 tumor-bearing mice treated with clodronate liposomes to deplete macrophages. Control mice received dimethyl sulfoxide. (I) The mRNA expression of CD8a, CCL5, CXCL9 and CXCL10 in tumor tissues after indicated treatments was determined by qRT-PCR. Error bars represent mean \pm SEM ( $n>7$ per group in $H)$. $P$ value was calculated by unpaired Student's t-test. $\left({ }^{\star} P<0.05,{ }^{\star \star} \mathrm{p}<0.01,{ }^{\star \star \star} \mathrm{p}<0.001\right)$. MSNs, mesoporous silica nanoparticles; PD-1, programmed cell death protein 1; FSC, forward scatter.

In transcriptional regulatory analysis, NF- $\mathrm{KB}$ complex accounted for the top two ranked transcriptional factors (figure 5D), indicating a leading regulation of NF- $\mathrm{\kappa B}$ to Ccl5-correlated genes. Likewise, NF-KB signaling pathway and its related pathways (such as TLR signaling pathway, TNF signaling pathway) were significantly enriched (figure 5E). These results reveal NF- $\mathrm{KB}$ signaling as the most potential regulatory pathway for the expression of $\mathrm{T}$ cell-recruitment chemokines.

To explore the influence of MSNs on these regulatory pathways, we compared the expression of genes in top 10 enriched pathways after different treatments. MSNs pretreatment, especially with $\alpha \mathrm{PD}-1$ therapy, markedly increased the overall expression of these genes (figure $5 \mathrm{~F}$ ), suggesting a potential activation induced by MSNs. From these top pathways, we further focused on NF-אB signaling and its upstream pathways, TLR pathway and TNF pathway, which also represent two typical receptors (TLRs and TNFRs) in macrophages. Compared with $\alpha \mathrm{PD}-1$ treatment alone, MSNs combined with $\alpha \mathrm{PD}-1$ significantly upregulated all these pathways (figure 5G). These results were confirmed in tumor tissues by western blot. As expected, the protein levels of TLR4 signaling (TLR4, MyD88, and TRAF6), TNFR1 signaling (TNFR1, 
A

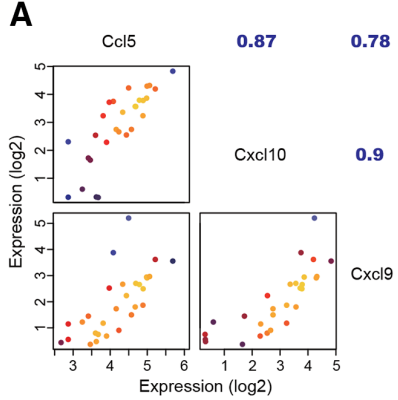

B

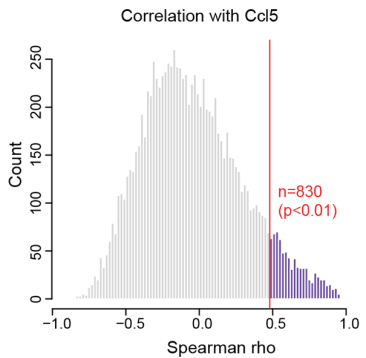

E

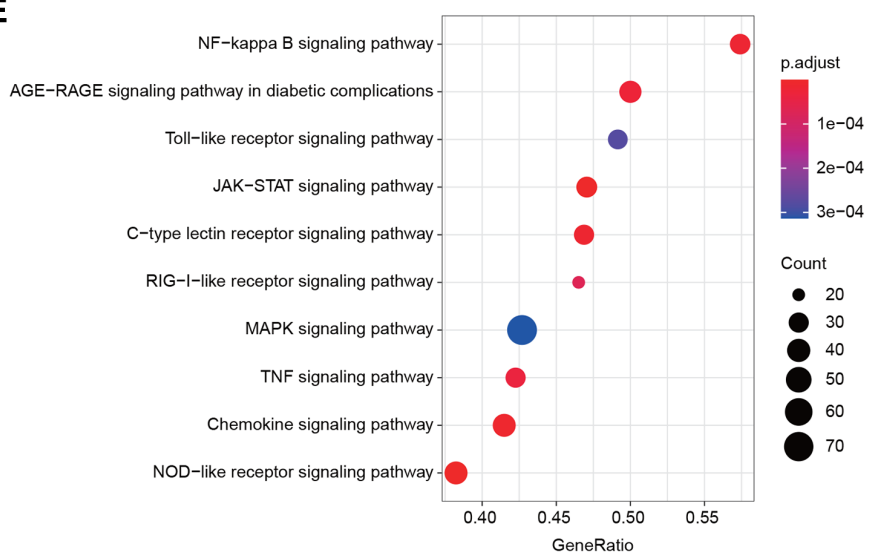

G

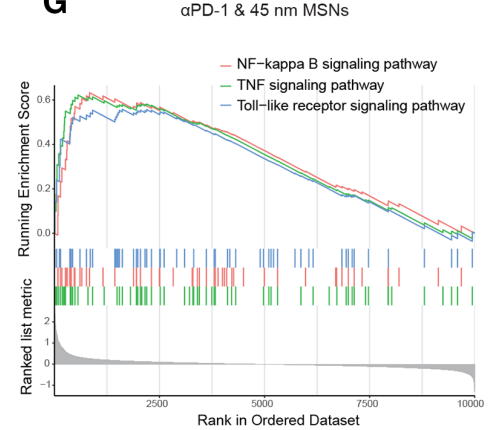

C
Cell-specific enrichment

D

Transcriptional regulatory enrichment
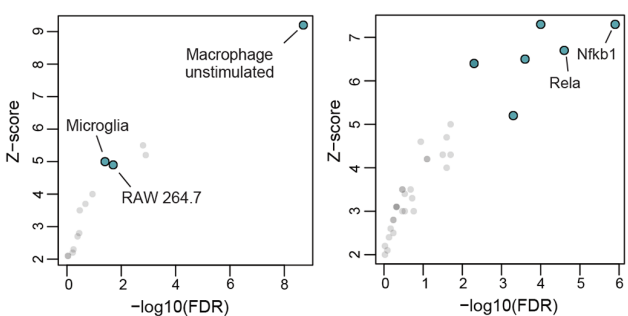

$\mathbf{F}$

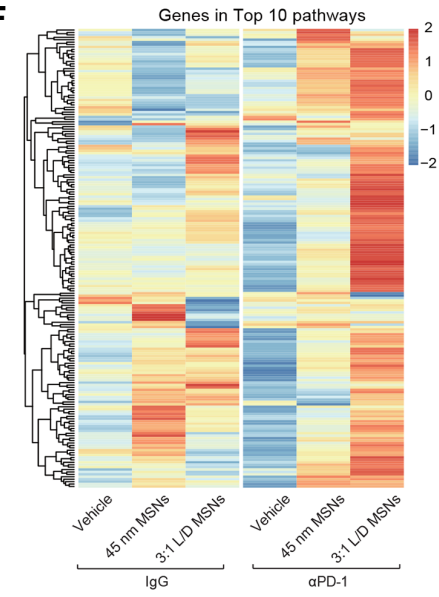

H

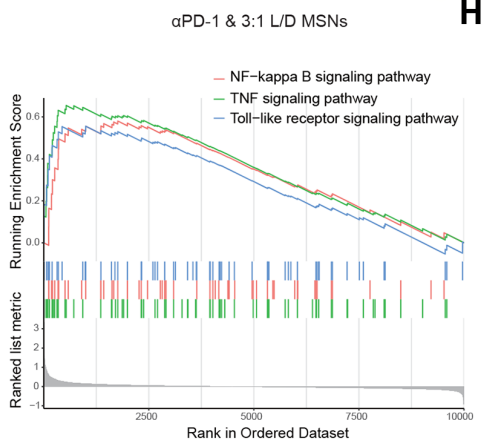

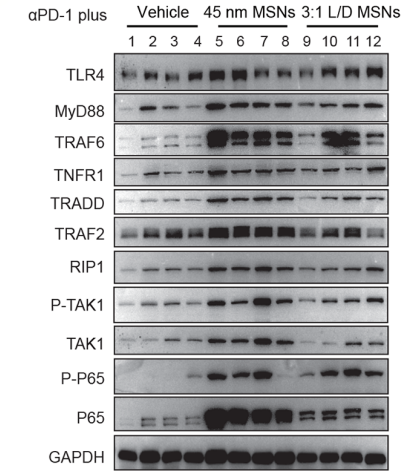

Figure 5 T cell-recruitment chemokines expression is associated with NF-KB signaling after MSNs treatment. (A) Correlation among the expression of T cell-recruitment chemokines across all B2m-sgRNA B16F10 tumor tissues. (B) Through transcriptomic analysis, all genes with significantly positive correlation with CCL5 were identified. (C) Cell-specific enrichment of CCL5-correlated genes. (D) Transcriptional regulatory enrichment of CCL5-correlated genes. (E) Top 10 signaling pathways by KEGG pathway enrichment of Ccl5-correlated genes. (F) In MSNs-treated tumors, the expression of genes in the top 10 Ccl5-related signaling pathways was presented by heatmap. (G) In tumor tissues, the activation changes of NF- $\mathrm{KB}$, TNF and TLR signaling pathways in $\alpha P D-1$ plus MSNs groups compared with $\alpha P D-1$ alone were revealed by GSEA. (H) In tumor tissues after indicated treatments, the levels of proteins in TLR4, TNFR and NF-KB signaling were determined by Western blot. GSEA, Gene Set Enrichment Analysis; MSNs, mesoporous silica nanoparticles; PD-1, programmed cell death protein 1; TLR, Toll-like receptor; TNF, tumor necrosis factor.

TRADD, TRAF2, and RIP1), as well as NF- $\mathrm{BB}$ signaling (phospho-P65) were enhanced in MSNs plus $\alpha \mathrm{PD}-1$ treatment (figure $5 \mathrm{H}$ ), suggesting that $\mathrm{NF}-\mathrm{\kappa B}$ and its upstream signaling pathways played key roles in the expression of $\mathrm{T}$ cell-recruitment chemokines after MSNs treatment.

\section{MSNs activate TLR4/NF-кB in macrophages to overcome tumor resistance}

We further explored how MSNs activated NF- $\mathrm{kB}$ pathway to overcome tumor resistance in vitro and in vivo. First, we examined the influence of MSNs in vitro and found that MSNs treatment markedly activated NF-KB signaling in Raw 264.7 cells (figure 6A). NF-אB signaling inhibition (JSH-23) in macrophages abrogated the promoting effect of MSNs on T cells recruitment. Following MSNs treatment, either macrophage-produced $\mathrm{T}$ cell-recruitment chemokines (figure 6B) or macrophage-recruited $\mathrm{T}$ cells (figure 6C) were dramatically compromised in NF-KB inhibited groups, suggesting that NF- $\mathrm{KB}$ signaling mediated MSNs-induced 

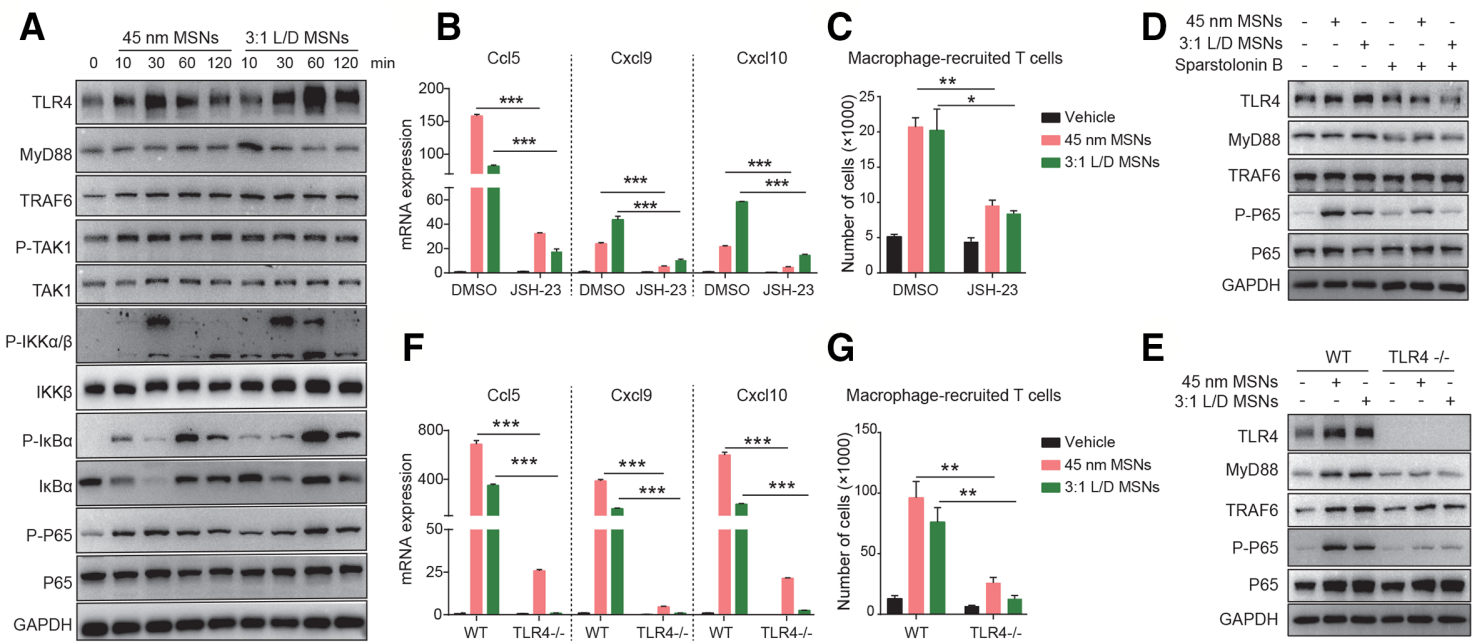

$\mathbf{F}$

G
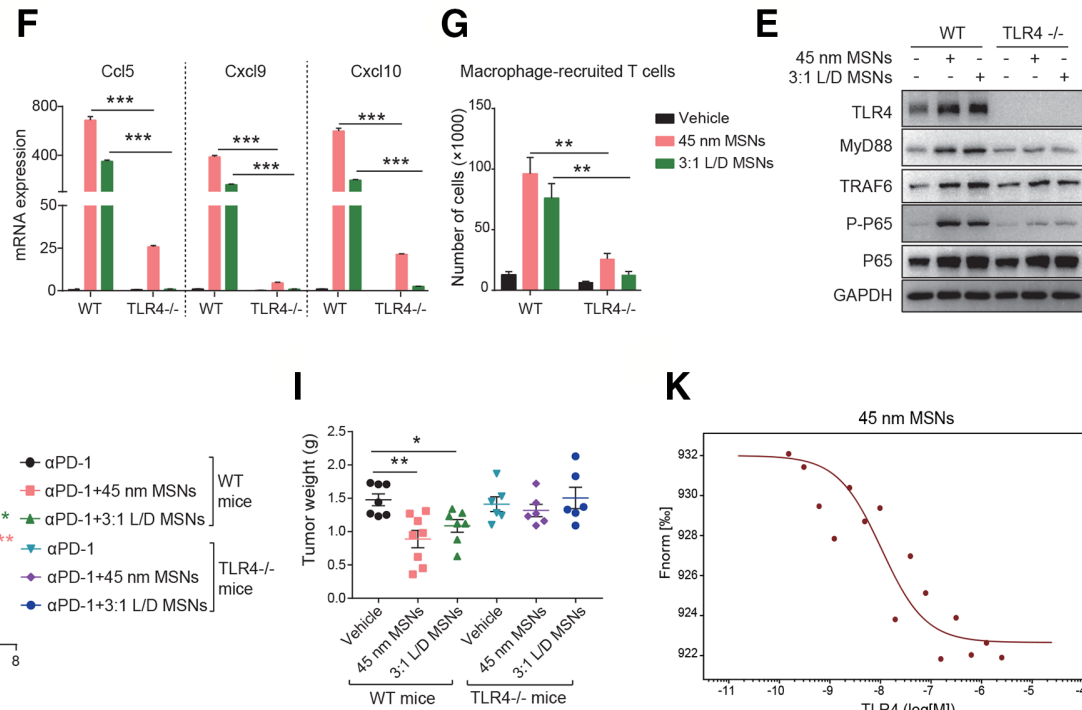

K
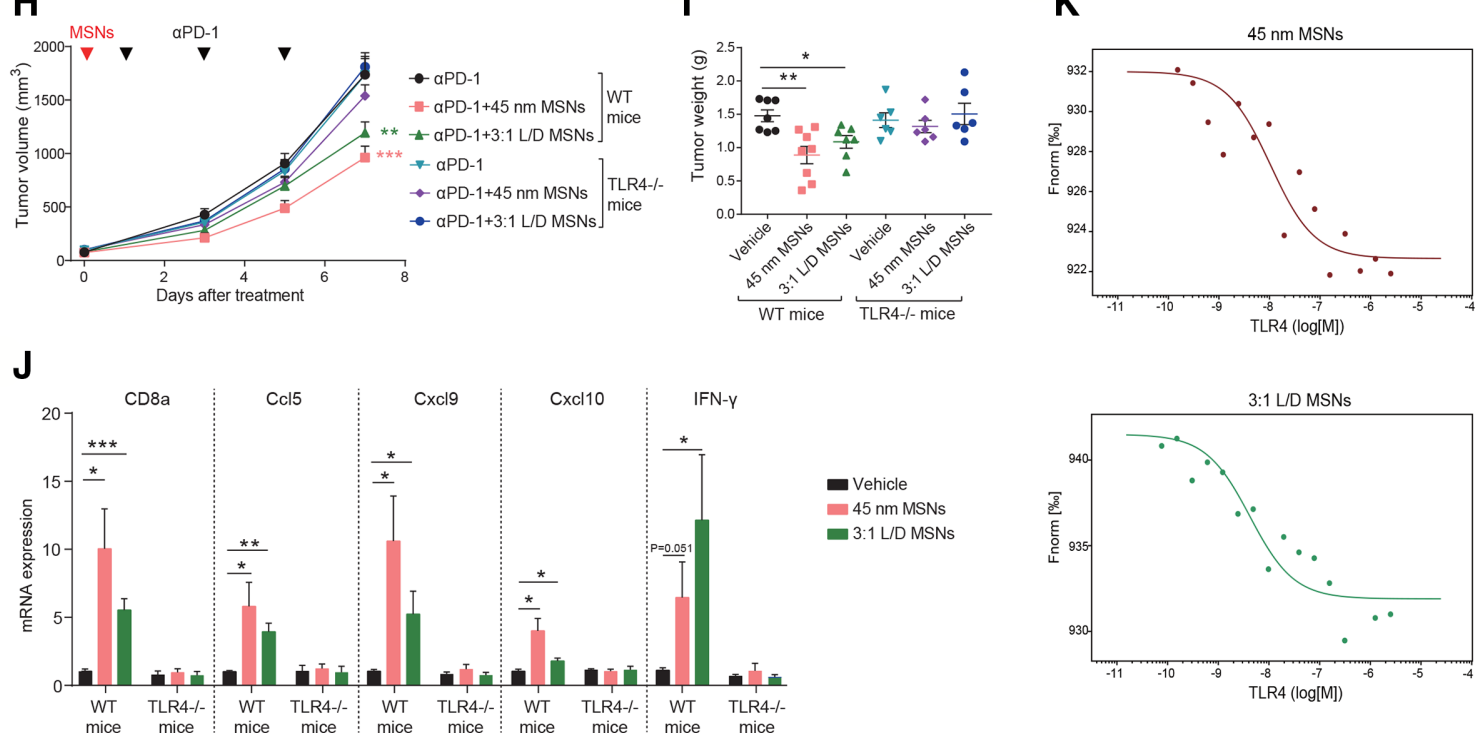

Figure 6 MSNs overcome tumor resistance through activating TLR4/NF-KB signaling in macrophages. (A) RAW 264.7 cells were treated with $100 \mu \mathrm{g} / \mathrm{mL}$ MSNs for different times and the protein levels were determined by Western blot. (B) RAW 264.7 cells were treated with $10 \mu \mathrm{M} \mathrm{JSH}-23$ for 3 hours and then $100 \mu \mathrm{g} / \mathrm{mL}$ MSNs for another 12 hours. the mRNA expression of CCL5, CXCL9 and CXCL10 was determined by qRT-PCR. (C) RAW 264.7 cells were treated with $10 \mu \mathrm{M} \mathrm{JSH}-23$ for 3 hours and then $100 \mu \mathrm{g} / \mathrm{mL}$ MSNs for another 12 hours. T cells recruited by the supernatant of macrophages were quantified through migration assay. (D) RAW 264.7 cells were treated with $100 \mu \mathrm{M}$ TLR4 blockade sparstolonin B for 3 hours and then $100 \mu \mathrm{g} /$ $\mathrm{mL}$ MSNs treatment for 1 hour. The protein levels were determined by Western blot. (E) Bone marrow-derived macrophages (BMDMs) from wide type (WT) and TLR4-deficient $\left(\mathrm{TLR}^{-/}{ }^{-}\right.$) mice were treated with $100 \mu \mathrm{g} / \mathrm{mL}$ MSNs for 1 hour. The protein levels were determined by Western blot. (F) WT and TLR4 $4^{-/-}$BMDMs were treated $100 \mu \mathrm{g} / \mathrm{mL}$ MSNs treatment for 12 hours. The mRNA expression of CCL5, CXCL9 and CXCL10 was determined by qRT-PCR. (G) WT and TLR4 ${ }^{-/-}$BMDMs were treated $100 \mu \mathrm{g} / \mathrm{mL}$ MSNs treatment for 12 hours. T cells recruited by the supernatant of macrophages were quantified through migration assay. $(\mathrm{H}, \mathrm{I})$ Tumor growth $(\mathrm{H})$ and tumor weights (I) of B2m-sgRNA B16F10 tumors in WT and TLR4 $4^{-/-}$mice after indicated treatments. (J) After indicated treatments, the mRNA expression of CD8a, CCL5, CXCL9, CXCL10 and IFN- $\gamma$ in tumor tissues was determined by qRT-PCR. Error bars represent mean \pm SEM $(n>5$ per group in $H)$. (K) The dose-response curves of MSNs with TLR4 in MST affinity analysis. The concentrations were $0.2 \mathrm{mg} / \mathrm{mL}$ for FITC-labeled MSNs and $0.1 \mathrm{nM}-2.5 \mu \mathrm{M}$ for TLR4 protein. $\mathrm{P}$ value was calculated by unpaired Student's t-test. $\left({ }^{*} \mathrm{P}<0.05,{ }^{* *} \mathrm{p}<0.01,{ }^{* * *} \mathrm{p}<0.001\right)$. MSNs, mesoporous silica nanoparticles; IFN- $\gamma$, interferon- $\gamma$; TLR4, Toll-like receptor 4.

$\mathrm{T}$ cells recruitment. Second, we identified the responsible receptor for MSNs-induced NF-KB activation. MSNs enhanced the protein levels of TLR4 pathway in Raw 264.7 cells (figure 6A). Further, TLR4 blockade (Sparstolonin B) and TLR4 deficiency (TLR4 $\left.4^{--}\right)$in macrophages prevented MSNs-induced activation of NF- $\mathrm{KB}$ (figure $6 \mathrm{D}, \mathrm{E}$ ), as well as macrophage-produced $\mathrm{T}$ cells recruitment (figure $6 \mathrm{~F}, \mathrm{G}$ ), suggesting that MSNs activated NF- $\mathrm{KB}$ in macrophages via TLR4. In the contrast, the protein levels of TNFR1 signaling were slightly affected by MSNs (online supplemental figure 9A). Anti-TNFR1 antibody (online supplemental figure 9B) and TNFR1 deficiency $\left(\mathrm{TNFR}^{-/}{ }^{-}\right.$) (online supplemental 


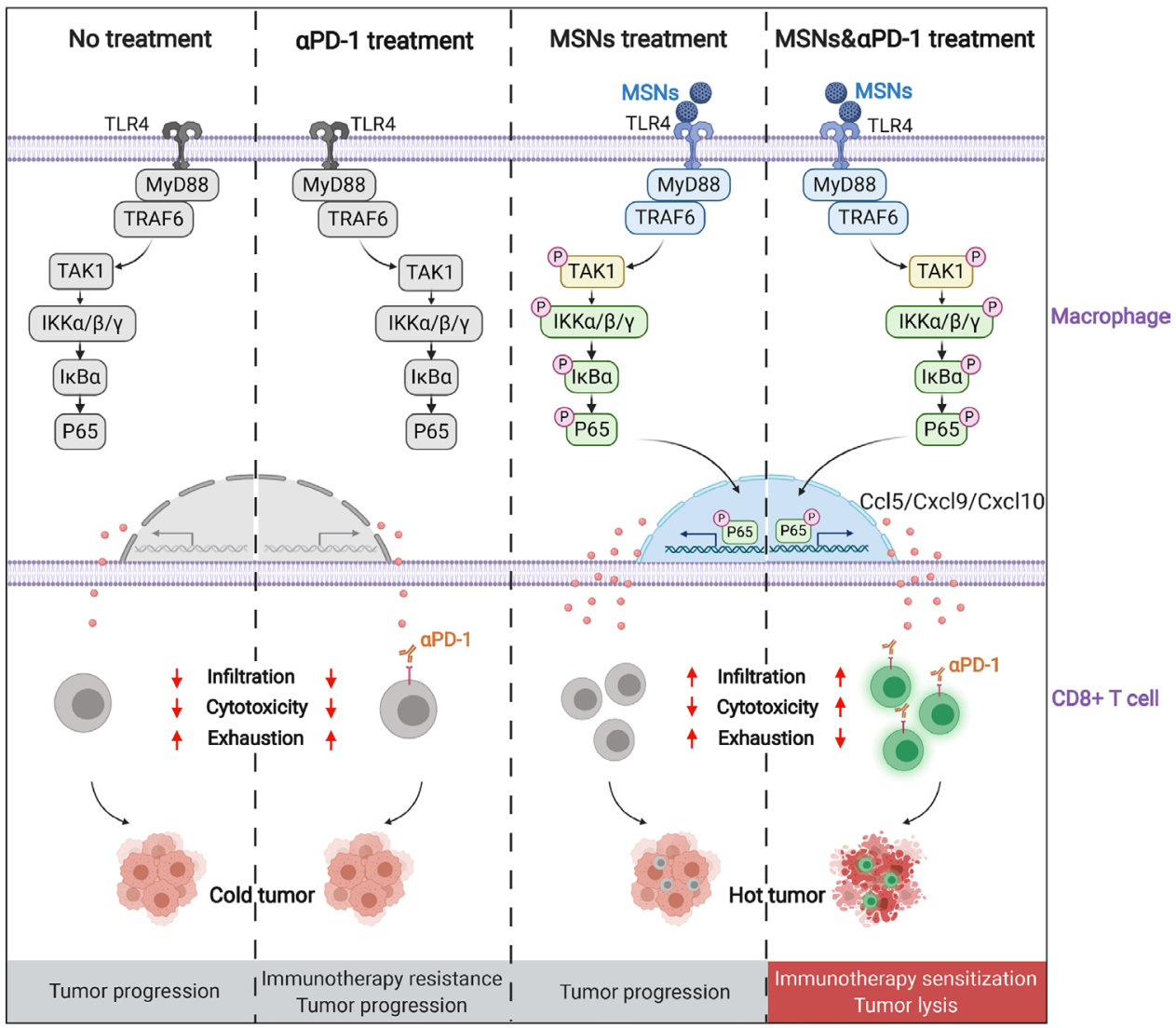

Figure 7 Schematic depiction of the synergistical effect of MSNs and PD-1 blockade on tumor immunotherapy. MSNs, mesoporous silica nanoparticles; PD-1, programmed cell death protein 1; TLR4, Toll-like receptor 4.

figure 9C-E) had little impact on NF-kB activation or T cells recruitment induced by MSNs. Finally, we determined whether MSNs overcame tumor resistance through TLR4/ $\mathrm{NF}-\mathrm{KB}$ pathway. In TLR4 ${ }^{--}$mice, the resensitizing effect of MSNs to $\alpha \mathrm{PD}-1$ treatment was completely abolished (figure $6 \mathrm{H}, \mathrm{I}$ ). Moreover, tumorous $\mathrm{CD}^{+} \mathrm{T}$ cells infiltration (CD8a), cytotoxicity (IFN- $\gamma$ ), and chemokines (Ccl5, Cxcl9, and Cxcl10) were not influenced by MSNs any more (figure 6J), confirming the essential role of TLR4 in MSNs-induced CTLs infiltration, activation and tumor resensitization.

To determine whether MSNs interact with TLR4 directly, we performed MST assay using purified TLR4 protein and FITC-labeled MSNs. As shown in figure 6K, we observed the variations of fluorescence signal along with the TLR4 concentration, suggesting a direct interaction between MSNs and TLR4. The dissociation constants $\left(\mathrm{K}_{\mathrm{D}}\right)$ of $45 \mathrm{~nm}$ MSNs and 3:1 L/D MSNs with TLR4 were calculated to be 10.5 and $4.2 \mathrm{nM}$, respectively, which representing the high binding affinity. ${ }^{21}$

Taken together, these results demonstrate that MSNs activate TLR4/NF- $\mathrm{KB}$ signaling in macrophages, promote $\mathrm{T}$ cell-recruitment chemokines production, drive CTLs tumorous infiltration, inflame the cold tumors and overcome the resistance to $\alpha$ PD-1 therapy (figure 7).

\section{DISCUSSION}

Our results demonstrate that nanomaterials themselves can overcome resistance to anti-PD-1 therapy. This effect is correlated with particle diameter and aspect ratio parameters. Even only once injection, potent therapeutic effects are invoked as early as the second day after antiPD-1 therapy, and immune memory persists until the end of treatment. By recruiting and activating CTLs, MSNs plus $\alpha \mathrm{PD}-1$ rapidly establish a T cell-inflamed TME and induce a long-lasting systemic antitumor immune response. Notably, as the representative nanomaterials, research on MSNs could shed light on the influences of physicochemical properties of materials on immunotherapy and improve the development and application of immunostimulant functional materials.

MSNs have been broadly explored in the biomedical field for two decades and represent remarkable biocompatibility, a critical consideration for clinical application. ${ }^{22}$ In vivo studies show that almost all MSNs $(94.4 \%)$ injected into mice were excreted out through urine and feces within 4 days. ${ }^{23}$ The US Food and Drug Administration (FDA) approved Cornell dots (C-dots) as the first silica-based tumor diagnostic nanoparticles for stage I human clinical trials in 2011, indicating biosafety and massive potential for clinical viability of silica-based nanoparticulate systems. In this study, despite MSNs triggering significant $\mathrm{T}$ cell activation and enhancing the 
immunotherapeutic effect of PD-1 blockade, no discernible differences in mouse body weight were observed among treatment groups, implying efficient and safe actions. Both granular and rod-shaped MSNs were found to overcome tumor resistances to anti-PD-1 therapy, with potency shown to be stronger in smaller size and aspect ratio. This should be considered in future development of immune-activating materials. Notably, although MSNs can enhance CTLs infiltration in vivo, MSNs pretreatment combined with IgG did not elicit therapeutic efficacy in vivo, suggesting that in our experimental settings (only injection once before the day of $\alpha \mathrm{PD}-1$ treatment), MSNs alone were not enough to exert potent antitumor effects. However, MSNs and $\alpha$ PD-1 exerted a synergistic effect on T cell function in terms of exhaustion and cytotoxicity, which subsequently promoted efficacy and overcame resistance to anti-PD-1 therapy.

The molecular mechanisms of tumor resistance to immunotherapy remain poorly understood. Known mechanisms of resistance to anti-PD-1 therapy involve tumor cell-intrinsic and tumor cell-extrinsic factors. ${ }^{3}$ Tumor cell-intrinsic factors include absence of antigen presentation and insensibility to $\mathrm{T}$ cells, whereas tumor cell-extrinsic mechanisms include decreased infiltration of CTLs, increased levels of immunosuppressive cells, and enhanced inhibitory immune checkpoints (eg, PD-1, LAG-3 and TIM-3) on CTLs. Our data demonstrated that MSNs rapidly established a T cell-inflamed TME, characterized by abundant high-activated and low-exhausted CTLs, which were further showed to be essential in eliciting a response to anti-PD-1 therapy, and immunotherapeutic efficacy will be entirely negated if CTLs infiltration is blocked. Sufficient inflammation-inducing immune cells can overcome resistance to anti-PD-1 therapy, even in the absence of antigen presentation. ${ }^{24}$ Interestingly, in our study, MSNs increased the proportion of non-major histocompatibility complex (MHC)-I-restricted immune cells (such as $\mathrm{CD}^{+}$T cells and NK) in $B 2 m$-sgRNA B16F10 tumor (figure 2A). One explanation is that $B 2 m-s g R N A$ B16F10 contained one-third of non-depleted cells due to multiple cell colonies, however, the potential roles of non-MHC-I-restricted immune cells cannot be completely excluded. Furthermore, our results showed $\mathrm{CD}^{+} \mathrm{T}$ cells are required for the resensitizing effect of MSNs. Therefore, whether the recognition-independent activities of $\mathrm{CD}^{+} \mathrm{T}$ cells are involved in the tumorous infiltration of other immune cells? To what extent do these immune cells contribute to the resistance reversal induced by MSNs? These questions are important and should be investigated in the future.

Chemokines play critical roles in $\mathrm{T}$ cell trafficking, which prompted us to investigate the chemokinecentric regulatory modules responsible for the recruitment of CTLs following MSNs treatment. Ccl5, Cxc19 and Cxcl10 in cancer have been reported to correlate with CTLs infiltration..$^{25}$ Our data indicated that macrophages accounted for the predominant source of Ccl5 and Cxc19 in tumors. Most nanomaterials after injection are rapidly eliminated by the mononuclear phagocyte system, whereas macrophages comprise the major cell population. ${ }^{26} 27$ Previous studies have determined that MSNs are preferentially internalized by macrophages in vivo, ${ }^{28}{ }^{29}$ suggesting that macrophages might be the main target cell of MSNs. Although the total proportion of macrophages in tumors did not always show increased trend following MSNs treatment, MSNs remarkably promoted the expression of Ccl5, Cxc19 and Cxcl10 in macrophages both in vitro and in vivo, and subsequently enhanced $\mathrm{CD}^{+} \mathrm{T}$ cells recruitment by macrophages, suggesting that MSNs modulate macrophages in terms of activation rather than amount.

TLR4 and NF-KB signaling has been identified as the target pathway for the influence of MSNs on macrophages. NF-KB is a well-characterized transcription factor involving in the transcriptional regulation of $\mathrm{T}$ cellrecruitment chemokines (Ccl5, Cxc19 and Cxcl10). ${ }^{30} 31$ There was a robust correlation between NF- $\mathrm{KB}$ signaling and $\mathrm{T}$ cell-recruitment chemokines in our data. Also, inhibition of NF- $\mathrm{BB}$ blocked MSNs-mediated T cell recruitment, suggesting that NF- $\mathrm{BB}$ signaling is responsible for CTLs tumorous infiltration under MSNs treatment. TLR4 was identified as a target of MSNs which further promoted NF- $\kappa B$ signaling. TLR4-based immunotherapy has been widely studied due to the crucial roles of TLR4 in both innate and adaptive immunity. ${ }^{32}$ TLR4 agonists, such as monophosphoryl lipid A, have been explored as potential adjuvants and obtained the FDA approval for clinical application in cancer therapy. In this study, MSNs upregulated TLR4, specifically triggered its downstream signaling in macrophages, and eventually overcame resistance to anti-PD-1 therapy, indicating that TLR4-based nanosystems could be considered as potential adjuvants for improving cancer immunotherapy. The size-relevant and morphology-relevant effects of MSNs on activating TLR4 in macrophages is worthy for further investigation in future studies.

\section{CONCLUSION}

In conclusion, this work demonstrates that MSNs trigger TLR4/NF-KB pathway in macrophages, drive the expression of $\mathrm{T}$ cell-recruitment chemokines, promote the tumorous infiltration of CTLs, and inflame immunologically cold tumors. By remodeling T cell-inflamed TME, MSNs plus PD-1 blockade overcome resistance to antiPD-1 therapy in diameter-dependent and aspect ratiodependent manners. This rational combination treatment efficiently inhibits tumor growth and recurrence with no obvious toxicity. This is the first report to demonstrate that biocompatible MSNs themselves (without additional functional transformation) can inflame cold tumors and overcome tumor resistance to anti-PD-1 therapy. Our findings provide rationale and preclinical evidence for the potential application of basic and enhanced functional MSNs in cancer immunotherapy. 


\section{Author affiliations}

${ }^{1}$ State Key Laboratory of Oncogenes and Related Genes, Center for Single-Cell Omics, School of Public Health, Shanghai Jiao Tong University School of Medicine, Shanghai, China

${ }^{2}$ CAS Key Laboratory of Nutrition, Metabolism and Food safety, Shanghai Institute of Nutrition and Health, University of Chinese Academy of Sciences, Chinese Academy of Sciences, Shanghai, China

${ }^{3}$ State Key Lab of High Performance Ceramics and Superfine Microstructure, Shanghai Institute of Ceramics, Chinese Academy of Sciences, Shanghai, China

Contributors MS, PG, YY, ZJ and JL performed all in vivo and in vitro experiments. $\mathrm{LY}$ synthesised and characterized all nanomaterials used in the study. MS, QB and YC analyzed the data. MS and QB wrote the manuscript. YC, QB and HW supervized the study.

Funding This study was supported by grants from the National Key R\&D Program of China (2018YFC2000700 and 2016YFA0203700), the National Natura Science Foundation of China $(82030099,81630086,81973078,51672303$, and 51722211), Shanghai Public Health System Construction Three-Year Action Plan (GWV-10.1-XK15), Shanghai Municipal Human Resources and Social Security Bureau (2018060), Program of Shanghai Subject Chief Scientist (18XD1404300), and the International Cooperation Project of Guangzhou Development Zone (2017GH11), Innovative research team of high-level local universities in Shanghai.

Competing interests None declared.

Patient consent for publication Not required.

Ethics approval The animal study was reviewed and approved by the Institutional Animal Care and Use Committee (IACUC) of the Institute for Nutritional Sciences.

Provenance and peer review Not commissioned; externally peer reviewed.

Data availability statement All data relevant to the study are included in the article or uploaded as online supplemental information.

Supplemental material This content has been supplied by the author(s). It has not been vetted by BMJ Publishing Group Limited (BMJ) and may not have been peer-reviewed. Any opinions or recommendations discussed are solely those of the author(s) and are not endorsed by BMJ. BMJ disclaims all liability and responsibility arising from any reliance placed on the content. Where the content includes any translated material, BMJ does not warrant the accuracy and reliability of the translations (including but not limited to local regulations, clinical guidelines, terminology, drug names and drug dosages), and is not responsible for any error and/or omissions arising from translation and adaptation or otherwise.

Open access This is an open access article distributed in accordance with the Creative Commons Attribution Non Commercial (CC BY-NC 4.0) license, which permits others to distribute, remix, adapt, build upon this work non-commercially, and license their derivative works on different terms, provided the original work is properly cited, appropriate credit is given, any changes made indicated, and the use is non-commercial. See http://creativecommons.org/licenses/by-nc/4.0/.

\section{ORCID iD}

Hui Wang http://orcid.org/0000-0003-2791-8981

\section{REFERENCES}

1 Sharpe $\mathrm{AH}$, Wherry EJ, Ahmed R, et al. The function of programmed cell death 1 and its ligands in regulating autoimmunity and infection. Nat Immunol 2007;8:239-45.

2 Wherry EJ, Kurachi M. Molecular and cellular insights into T cell exhaustion. Nat Rev Immunol 2015;15:486-99.

3 Sharma P, Hu-Lieskovan S, Wargo JA, et al. Primary, adaptive, and acquired resistance to cancer immunotherapy. Cell 2017;168:707-23.

4 Reck M, Rodríguez-Abreu D, Robinson AG, et al. Pembrolizumab versus chemotherapy for PD-L1-positive non-small-cell lung cancer. N Engl J Med 2016;375:1823-33.

5 Bonaventura P, Shekarian T, Alcazer V, et al. Cold tumors: a therapeutic challenge for immunotherapy. Front Immunol 2019;10:168.

6 Mahoney KM, Rennert PD, Freeman GJ. Combination cancer immunotherapy and new immunomodulatory targets. Nat Rev Drug Discov 2015;14:561-84.
7 Salata OV. Applications of nanoparticles in biology and medicine. J Nanobiotechnology 2004;2:3.

8 Wei M, Li S, Le W. Nanomaterials modulate stem cell differentiation: biological interaction and underlying mechanisms.

J Nanobiotechnology 2017;15:75.

9 Li X, Liu W, Sun L, et al. Effects of physicochemical properties of nanomaterials on their toxicity. J Biomed Mater Res A 2015;103:2499-507.

10 Lehner R, Wang X, Marsch S, et al. Intelligent nanomaterials for medicine: carrier platforms and targeting strategies in the context of clinical application. Nanomedicine 2013;9:742-57.

11 Tarn D, Ashley CE, Xue M, et al. Mesoporous silica nanoparticle nanocarriers: biofunctionality and biocompatibility. Acc Chem Res 2013;46:792-801.

12 Wang Y, Zhao Q, Han N, et al. Mesoporous silica nanoparticles in drug delivery and biomedical applications. Nanomedicine 2015;11:313-27.

13 Kupferschmidt N, Qazi KR, Kemi C, et al. Mesoporous silica particles potentiate antigen-specific T-cell responses. Nanomedicine 2014:9:1835-46.

14 Pang S, Tang $\mathrm{H}$, Zhuo S, et al. Regulation of fasting fuel metabolism by Toll-like receptor 4. Diabetes 2010;59:3041-8.

15 Zhou Y, Zhou B, Pache L, et al. Metascape provides a biologistoriented resource for the analysis of systems-level datasets. Nat Commun 2019;10:1523.

16 Liu H, Shen J, Lu K. II-6 and PD-L1 blockade combination inhibits hepatocellular carcinoma cancer development in mouse model. Biochem Biophys Res Commun 2017;486:239-44.

17 Ho WS, Wang H, Maggio D, et al. Pharmacologic inhibition of protein phosphatase-2A achieves durable immune-mediated antitumor activity when combined with PD-1 blockade. Nat Commun 2018;9:2126.

18 Mombaerts P, lacomini J, Johnson RS, et al. RAG-1-deficient mice have no mature B and T lymphocytes. Cell 1992;68:869-77.

19 House IG, Savas P, Lai J, et al. Macrophage-Derived CXCL9 and CXCL10 are required for antitumor immune responses following immune checkpoint blockade. Clin Cancer Res 2020;26:487-504

20 Yang Y, Sun M, Yao W, et al. Compound kushen injection relieves tumor-associated macrophage-mediated immunosuppression through TNFR1 and sensitizes hepatocellular carcinoma to sorafenib. $\mathrm{J}$ Immunother Cancer 2020;8:e000317.

21 Cao Z-T, Gan L-Q, Jiang W, et al. Protein binding affinity of polymeric nanoparticles as a direct indicator of their pharmacokinetics. ACS Nano 2020;14:3563-75.

22 Nguyen TL, Choi Y, Kim J. Mesoporous silica as a versatile platform for cancer immunotherapy. Adv Mater 2019;31:e1803953:1803953.

$23 \mathrm{Lu} \mathrm{J}$, Liong M, Li Z, et al. Biocompatibility, biodistribution, and drugdelivery efficiency of mesoporous silica nanoparticles for cancer therapy in animals. Small 2010;6:1794-805.

24 Ishizuka JJ, Manguso RT, Cheruiyot CK, et al. Loss of ADAR1 in tumours overcomes resistance to immune checkpoint blockade. Nature 2019;565:43-8.

25 Harlin $\mathrm{H}$, Meng Y, Peterson AC, et al. Chemokine expression in melanoma metastases associated with CD8+ T-cell recruitment. Cancer Res 2009;69:3077-85

26 Kwon D, Cha BG, Cho Y, et al. Extra-Large pore mesoporous silica nanoparticles for directing in vivo M2 macrophage polarization by delivering IL-4. Nano Lett 2017;17:2747-56.

27 Fu C, Liu T, Li L, et al. The absorption, distribution, excretion and toxicity of mesoporous silica nanoparticles in mice following different exposure routes. Biomaterials 2013;34:2565-75.

28 van Rijt SH, Bölükbas DA, Argyo C, et al. Applicability of avidin protein coated mesoporous silica nanoparticles as drug carriers in the lung. Nanoscale 2016;8:8058-69.

29 Hoppstädter J, Seif M, Dembek A, et al. M2 polarization enhances silica nanoparticle uptake by macrophages. Front Pharmacol 2015;6:55.

30 Wickremasinghe MI, Thomas LH, O'Kane CM, et al. Transcriptional mechanisms regulating alveolar epithelial cellspecific CCL5 secretion in pulmonary tuberculosis. J Biol Chem 2004;279:27199-210.

31 Hiroi M, Ohmori Y. Constitutive nuclear factor kappaB activity is required to elicit interferon-gamma-induced expression of chemokine CXC ligand 9 (CXCL9) and CXCL10 in human tumour cell lines. Biochem J 2003;376:393-402.

32 Shetab Boushehri MA, Lamprecht A. TLR4-Based immunotherapeutics in cancer: a review of the achievements and shortcomings. Mol Pharm 2018;15:4777-800. 